\title{
Fast and accurate predictions of covalent bonds in chemical space
}

K. Y. Samuel Chang, Stijn Fias, Raghunathan Ramakrishnan, and O. Anatole von Lilienfeld'

Citation: The Journal of Chemical Physics 144, 174110 (2016); doi: 10.1063/1.4947217

View online: http://dx.doi.org/10.1063/1.4947217

View Table of Contents: http://aip.scitation.org/toc/jcp/144/17

Published by the American Institute of Physics

\section{Articles you may be interested in}

Communication: Understanding molecular representations in machine learning: The role of uniqueness and target similarity

The Journal of Chemical Physics 145, 161102161102 (2016); 10.1063/1.4964627

Guiding ab initio calculations by alchemical derivatives

The Journal of Chemical Physics 144, 104103104103 (2016); 10.1063/1.4943372

Electronic spectra from TDDFT and machine learning in chemical space

The Journal of Chemical Physics 143, 084111084111 (2015); 10.1063/1.4928757

Accurate ab initio energy gradients in chemical compound space

The Journal of Chemical Physics 131, 164102164102 (2009); 10.1063/1.3249969

Tuning dissociation using isoelectronically doped graphene and hexagonal boron nitride: Water and other small molecules

The Journal of Chemical Physics 144, 154706154706 (2016); 10.1063/1.4945783

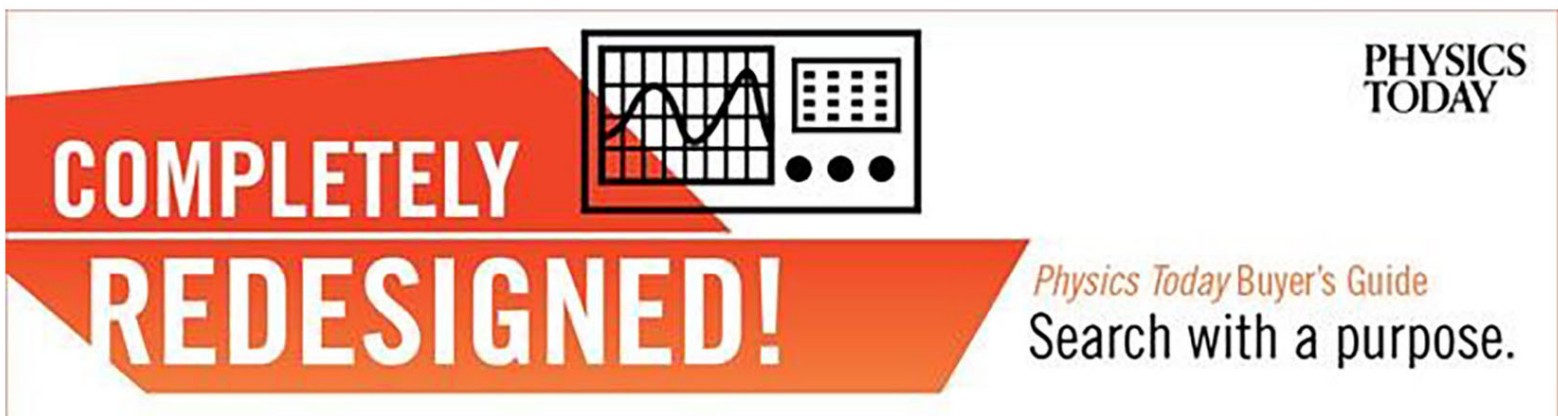




\title{
Fast and accurate predictions of covalent bonds in chemical space
}

\author{
K. Y. Samuel Chang, ${ }^{1}$ Stijn Fias, ${ }^{2}$ Raghunathan Ramakrishnan, ${ }^{1}$ \\ and $\mathrm{O}$. Anatole von Lilienfeld ${ }^{1,2, a)}$ \\ ${ }^{1}$ Department of Chemistry, Institute of Physical Chemistry and National Center for Computational Design \\ and Discovery of Novel Materials (MARVEL), University of Basel, 4056 Basel, Switzerland \\ ${ }^{2}$ General Chemistry (ALGC), Free University Brussels (VUB), Pleinlaan 2, 1050 Brussels, Belgium
}

(Received 12 January 2016; accepted 8 April 2016; published online 4 May 2016)

\begin{abstract}
We assess the predictive accuracy of perturbation theory based estimates of changes in covalent bonding due to linear alchemical interpolations among molecules. We have investigated $\sigma$ bonding to hydrogen, as well as $\sigma$ and $\pi$ bonding between main-group elements, occurring in small sets of iso-valence-electronic molecules with elements drawn from second to fourth rows in the $p$-block of the periodic table. Numerical evidence suggests that first order Taylor expansions of covalent bonding potentials can achieve high accuracy if (i) the alchemical interpolation is vertical (fixed geometry), (ii) it involves elements from the third and fourth rows of the periodic table, and (iii) an optimal reference geometry is used. This leads to near linear changes in the bonding potential, resulting in analytical predictions with chemical accuracy $(\sim 1 \mathrm{kcal} / \mathrm{mol})$. Second order estimates deteriorate the prediction. If initial and final molecules differ not only in composition but also in geometry, all estimates become substantially worse, with second order being slightly more accurate than first order. The independent particle approximation based second order perturbation theory performs poorly when compared to the coupled perturbed or finite difference approach. Taylor series expansions up to fourth order of the potential energy curve of highly symmetric systems indicate a finite radius of convergence, as illustrated for the alchemical stretching of $\mathrm{H}_{2}^{+}$. Results are presented for (i) covalent bonds to hydrogen in 12 molecules with 8 valence electrons $\left(\mathrm{CH}_{4}, \mathrm{NH}_{3}, \mathrm{H}_{2} \mathrm{O}, \mathrm{HF}, \mathrm{SiH}_{4}, \mathrm{PH}_{3}, \mathrm{H}_{2} \mathrm{~S}, \mathrm{HCl}, \mathrm{GeH}_{4}\right.$, $\mathrm{AsH}_{3}, \mathrm{H}_{2} \mathrm{Se}, \mathrm{HBr}$ ); (ii) main-group single bonds in 9 molecules with 14 valence electrons $\left(\mathrm{CH}_{3} \mathrm{~F}\right.$, $\mathrm{CH}_{3} \mathrm{Cl}, \mathrm{CH}_{3} \mathrm{Br}, \mathrm{SiH}_{3} \mathrm{~F}, \mathrm{SiH}_{3} \mathrm{Cl}, \mathrm{SiH}_{3} \mathrm{Br}, \mathrm{GeH}_{3} \mathrm{~F}, \mathrm{GeH}_{3} \mathrm{Cl}, \mathrm{GeH} 3 \mathrm{Br}$ ); (iii) main-group double bonds in 9 molecules with 12 valence electrons $\left(\mathrm{CH}_{2} \mathrm{O}, \mathrm{CH}_{2} \mathrm{~S}, \mathrm{CH}_{2} \mathrm{Se}, \mathrm{SiH}_{2} \mathrm{O}, \mathrm{SiH}_{2} \mathrm{~S}, \mathrm{SiH}_{2} \mathrm{Se}, \mathrm{GeH}_{2} \mathrm{O}, \mathrm{GeH}_{2} \mathrm{~S}\right.$, $\mathrm{GeH}_{2} \mathrm{Se}$ ); (iv) main-group triple bonds in 9 molecules with 10 valence electrons (HCN, HCP, HCAs, HSiN, HSiP, HSiAs, HGeN, HGeP, HGeAs); and (v) $\mathrm{H}_{2}^{+}$single bond with 1 electron. Published by AIP Publishing. [http://dx.doi.org/10.1063/1.4947217]
\end{abstract}

\section{INTRODUCTION}

Solving Schrödinger's time independent equation for the unperturbed electronic ground-state within the BornOppenheimer approximation yields the potential energy surface (PES) of any molecule as a function of nuclear charges $\left\{Z_{I}\right\}$ (stoichiometry), nuclear positions $\left\{\mathbf{R}_{I}\right\}$ (geometry), and number of electrons $N$ (molecular charge). ${ }^{1,2}$ The PES plays a fundamental role in chemistry and elsewhere because many properties can be derived from it. While one can study efficient ways of predicting the PES of single compounds, ${ }^{3-5}$ efficient estimates of PES of ensembles of molecules are more useful (and challenging) in the context of virtual compound design efforts. ${ }^{6-10}$ These efforts typically attempt to search chemical compound space (CCS) spanned by $\left\{\left\{Z_{I}\right\},\left\{\mathbf{R}_{I}\right\}, N\right\}^{11,12}$ for novel materials with desirable properties. As such, accurate yet efficient quantum mechanics (QM) based PES estimates hold the key for successful rational compound design applications. ${ }^{6,7,13-15}$ While many inexpensive semi-empirical QM methods are available, for this study we restrict ourselves to first principles in the spirit of Refs. 12 and 16-22. More specifically, we investigate the application of "alchemical"

\footnotetext{
a)anatole.vonlilienfeld@unibas.ch
}

coupling to the problem of efficiently estimating the PES of new molecules using Taylor series expansions in CCS, rather than empiricism.

The alchemical coupling approach can be related to grand-

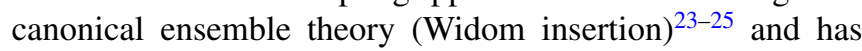
been well established for empirical force-field based molecular dynamics studies. ${ }^{26-30}$ Using QM, alchemical changes are less common despite E. B. Wilson's early proposal of variable $Z$, back in $1962 .{ }^{31}$ Within QM, any two iso-electronic molecules in CCS can be coupled "alchemically" through interpolation of their external potentials. Here, we have investigated if alchemical predictions can be used to model the PES of covalent bonds occurring in small closed-shell molecules made up from main group elements. We have limited ourselves to covalent bonds to hydrogen, as well as single, double, and triple bonds in molecules with no more than 14 valence electrons. We present and discuss numerical evidence for the following set of observations: First order Taylor-expansions of covalent bonding potentials can reach chemical accuracy $(\sim 1 \mathrm{kcal} / \mathrm{mol})$ if three conditions are met. First, the alchemical change has to be "vertical," meaning that initial reference molecule as well as final target molecule have to possess the same number of atoms located at the exact same positions. Second, all elements involved in the alchemical change, i.e., all 
$\left\{Z_{I}\right\}$ destined to vary, have to occur late in the periodic table. Third, the off-set of the bond potential, determined by reference distance, is optimized. Second order Taylorexpansion based predictions are less accurate than first order predictions if these conditions are met. If reference and target molecules have different geometries, the predictive power of the first order Taylor expansion substantially deteriorates, while second order estimates based on coupled perturbed (CP) Kohn-Sham equations offer some improvement, however, without reaching chemical accuracy. Second order estimates based on the independent particle approximation (IPA) result in Taylor expansion estimates that are even worse than first order estimates. For highly alchemical changes with symmetric endpoints, such as the dissociation of $\mathrm{H}_{2}^{+}$, a finite radius of convergence is found.

In Sec. II we briefly summarize the framework of alchemical derivatives within Hartree-Fock and density functional theory (DFT) as well as our notations. Numerical estimates of covalent bond stretching energies of small molecules are presented and discussed in Sec. III: Extending previous work on alchemical perturbation, ${ }^{19,20,32}$ we discuss alchemical energy derivatives with respect to vertical transmutation, interpolating only the identity of the atoms while keeping the geometry fixed. Estimates for single, double, and triple bonds are included as an application. We also report numerical results for alchemical stretching of chemical bonds using non-vertical transmutations. Conclusions are drawn in Sec. IV.

\section{METHOD}

\section{A. Taylor expansion in CCS}

A Taylor expansion of the potential energy in CCS can be constructed with the exclusive knowledge acquired by solving Schrödinger's equation for some reference molecule, with Hamiltonian $H_{\mathrm{R}}$,

$$
E(\Delta \lambda)=E_{\mathrm{R}}+\left.\Delta \lambda \partial_{\lambda} E_{\lambda}\right|_{\lambda=0}+\left.\frac{\Delta \lambda^{2}}{2} \partial_{\lambda}^{2} E_{\lambda}\right|_{\lambda=0}+\cdots
$$

Derivatives of the total potential energy can be obtained by coupling a reference Hamiltonian to some target Hamiltonian, $H_{\mathrm{T}}$, such that $H_{\lambda}$ transforms $H_{\mathrm{R}}$ into $H_{\mathrm{T}}$,

$$
H_{\lambda}=(1-\lambda) H_{\mathrm{R}}+\lambda H_{\mathrm{T}},
$$

as the coupling parameter $\lambda$ goes from 0 to 1 . And consequently, $\partial_{\lambda}^{m} E_{\lambda}=\partial_{\lambda}^{m}\left\langle H_{\lambda}\right\rangle$, with $\partial_{\lambda} H_{\lambda}=H_{\mathrm{T}}-H_{\mathrm{R}}=H^{\prime}$ being the alchemical perturbing Hamiltonian. If these derivatives can be computed, $E_{\mathrm{T}}$ can be estimated according to Eq. (1) by setting $\Delta \lambda=1$. Note that we couple reference and target systems in a linear and global fashion. This is an arbitrary choice; non-linear and local interpolation functions could have been chosen just as well. In fact, in Ref. 20, an empirical quadratic interpolation function is found to yield superior results for first order predictions of highest occupied molecular orbital (HOMO) eigenvalues. In this study of alchemical changes of covalent bonding, we begin with linear and global interpolations; future work might deal with alternative functions.
Given a pair of iso-electronic reference/target systems, described by $\left\{\left\{Z_{I}^{\mathrm{R}}\right\},\left\{\mathbf{R}_{I}^{\mathrm{R}}\right\}, N\right\}$ and $\left\{\left\{Z_{I}^{\mathrm{T}}\right\},\left\{\mathbf{R}_{I}^{\mathrm{T}}\right\}, N\right\}$, respectively, one can couple the two systems such that certain $Z_{I}^{\mathrm{R}}$ and $Z_{I}^{\mathrm{T}}$ are paired. Note that $Z_{I}^{\mathrm{R}}$ or $Z_{I}^{\mathrm{T}}$ can be scaled down to/up from zero if the number of atoms in one molecule is smaller. Under iso-electronic conditions, the $\lambda$-dependent terms in the coupling Hamiltonian (Eq. (2)) are the electron-nucleus and nucleus-nucleus interaction operators,

$$
\begin{aligned}
v_{\lambda}(\mathbf{r}) & =\sum_{I}^{N_{I}}\left(-\frac{(1-\lambda) Z_{I}^{\mathrm{R}}}{\left|\mathbf{r}-\mathbf{R}_{I}^{\mathrm{R}}\right|}-\frac{\lambda Z_{I}^{\mathrm{T}}}{\left|\mathbf{r}-\mathbf{R}_{I}^{\mathrm{T}}\right|}\right), \\
V_{\lambda} & =\sum_{I<J}^{N_{I}}\left(\frac{(1-\lambda) Z_{I}^{\mathrm{R}} Z_{J}^{\mathrm{R}}}{\left|\mathbf{R}_{I}^{\mathrm{R}}-\mathbf{R}_{J}^{\mathrm{R}}\right|}+\frac{\lambda Z_{I}^{\mathrm{T}} Z_{J}^{\mathrm{T}}}{\left|\mathbf{R}_{I}^{\mathrm{T}}-\mathbf{R}_{J}^{\mathrm{T}}\right|}\right) .
\end{aligned}
$$

Since different pairing schemes result in different $v_{\lambda}(\mathbf{r})$ and $V_{\lambda}$, it is obvious that the alchemical perturbation is alignment dependent. To investigate the behaviour of higher order corrections and the effects of varying geometry/stoichiometry, we neglect all relaxation effects for vertical iso-valenceelectronic changes (see Sec. II G).

\section{B. First order derivative}

The first order derivative of the energy with respect to an alchemical interpolation parameter connecting any two iso-electronic molecules can be computed according to the Hellmann-Feynman theorem, ${ }^{33}$ as has been shown for molecular HOMO eigenvalues, ${ }^{20}$

$$
\partial_{\lambda} E_{\lambda}=\left\langle\partial_{\lambda} H_{\lambda}\right\rangle_{\lambda}=\int d \mathbf{r} \rho_{\lambda}(\mathbf{r}) \partial_{\lambda} v_{\lambda}(\mathbf{r})+\partial_{\lambda} V_{\lambda},
$$

where $\rho_{\lambda}(\mathbf{r})$ denotes the electron density, dependent on $\lambda$. At $\lambda=0$ we have $\rho_{\lambda}(\mathbf{r})=\rho_{\mathrm{R}}(\mathbf{r})$, which is independent of the target system. As such, the first order derivative can be calculated with a single reference density and without additional self-consistent field (SCF) calculation for any target system. In several circumstances, Taylor expansion estimates using first order alchemical derivatives have shown good accuracy for the rapid prediction of properties throughout CCS. ${ }^{12,21,32,34,35}$ In general, however, first order derivatives might not be sufficient. Taking higher order derivatives into account might offer higher accuracy, assuming Eq. (1) converges rapidly.

\section{Second order derivative}

Differentiation of Eq. (4), based on linear interpolated Hamiltonian in Eq. (2), yields

$$
\partial_{\lambda}^{2} E_{\lambda}=\int d \mathbf{r}\left(\partial_{\lambda} \rho(\mathbf{r})\right)\left(\partial_{\lambda} v_{\lambda}(\mathbf{r})\right)
$$

requiring the density response due to the alchemical perturbation. Again, at $\lambda=0$ this amounts to the density response of the reference system. Evaluation of Eq. (5) implies a differing density response for each target system. We have considered three approximations to $\partial_{\lambda} \rho$ including second order perturbation theory with IPA, ${ }^{36} \mathrm{CP}$ approaches,,${ }^{37,38}$ as well as finite difference (FD) approximation. Note that Eq. (5) 
can be rewritten as $\partial_{\lambda}^{2} E=\int d \mathbf{r} d \mathbf{r}^{\prime}\left(\partial_{\lambda} v(\mathbf{r})\right)\left(\partial_{\lambda} v\left(\mathbf{r}^{\prime}\right)\right) \frac{\delta^{2} E}{\delta v(\mathbf{r}) \delta v\left(\mathbf{r}^{\prime}\right)}$, where $\frac{\delta^{2} E}{\delta v(\mathbf{r}) \delta v\left(\mathbf{r}^{\prime}\right)}=\chi\left(\mathbf{r}, \mathbf{r}^{\prime}\right)$ is the static linear response function or susceptibility, well established within conceptual DFT. ${ }^{39-44}$

Perturbation theory provides ways to estimate $\partial_{\lambda} \rho_{\lambda}(\mathbf{r}){ }^{45}$ Within IPA, ${ }^{36,46,47}$ the static density response for a close-shell system is approximated by

$\partial_{\lambda} \rho_{\lambda}(\mathbf{r}) \approx-4 \sum_{i a} \phi_{i}(\mathbf{r}) \phi_{a}(\mathbf{r}) \int d \mathbf{r}^{\prime} \frac{\phi_{i}\left(\mathbf{r}^{\prime}\right) \phi_{a}\left(\mathbf{r}^{\prime}\right)}{\varepsilon_{a}-\varepsilon_{i}} \partial_{\lambda} v_{\lambda}\left(\mathbf{r}^{\prime}\right)$,

where $\left\{\phi_{i}, \varepsilon_{i}\right\}$ denote the $i$ th occupied molecular orbital (MO) and its eigenvalue, while $\left\{\phi_{a}, \varepsilon_{a}\right\}$ denote the $a$ th unoccupied counterparts. IPA neglects the influence of the alchemical perturbation on the Hartree and exchange-correlation (xc) potentials. ${ }^{38,42}$ Note that Eq. (6) becomes numerically exact for 1-electron system with converged basis set within HartreeFock approximation because of the absence of Coulomb and $\mathrm{xc}$ interaction between electrons.

Recently, Yang, Cohen, De Proft, and Geerlings derived an expression of the density response that also includes the dependence of Coulomb and xc potential, ${ }^{37}$ the $\mathrm{CP}$ approach, ${ }^{44}$

$$
\begin{aligned}
\partial_{\lambda} \rho_{\lambda}(\mathbf{r})= & -4 \sum_{i j} \sum_{a b} \phi_{i}(\mathbf{r}) \phi_{a}(\mathbf{r}) \\
& \times\left(\mathbf{M}^{-1}\right)_{i a, j b} \int d \mathbf{r}^{\prime} \phi_{j}\left(\mathbf{r}^{\prime}\right) \phi_{b}\left(\mathbf{r}^{\prime}\right) \partial_{\lambda} v_{\lambda}\left(\mathbf{r}^{\prime}\right),
\end{aligned}
$$

where the matrix elements of $\mathbf{M}$ are

$$
\left\{\begin{aligned}
\mathbf{M}_{i a, j b} & =\left(\varepsilon_{a}-\varepsilon_{i}\right) \delta_{i j} \delta_{a b}+4 \mathbf{J}_{i a, j b}+4 \mathbf{X}_{i a, j b} \\
\mathbf{J}_{i a, j b} & =\int d \mathbf{r} d \mathbf{r}^{\prime} \frac{\phi_{i}(\mathbf{r}) \phi_{a}(\mathbf{r}) \phi_{j}\left(\mathbf{r}^{\prime}\right) \phi_{b}\left(\mathbf{r}^{\prime}\right)}{\left|\mathbf{r}-\mathbf{r}^{\prime}\right|} \\
\mathbf{X}_{i a, j b} & =\int d \mathbf{r} d \mathbf{r}^{\prime} \phi_{i}(\mathbf{r}) \phi_{a}(\mathbf{r}) \phi_{j}\left(\mathbf{r}^{\prime}\right) \phi_{b}\left(\mathbf{r}^{\prime}\right)\left(\frac{\delta^{2} E_{x c}}{\delta \rho(\mathbf{r}) \delta \rho\left(\mathbf{r}^{\prime}\right)}\right)
\end{aligned}\right.
$$

In the limit of $\mathbf{J}_{i a, j b} \rightarrow 0$ and $\mathbf{X}_{i a, j b} \rightarrow 0$, Eqs. (6) and (7) are equivalent. In our implementation, the evaluation of the $\mathrm{CP}$ second order derivative has a computational complexity similar to a molecular frequency calculation. IPA incurs negligible computational overhead.

Alternatively, one can also introduce an explicit small perturbation and converge the new density at $\Delta \lambda \ll 1$. The density response can then be estimated via $\mathrm{FD}, \partial_{\lambda} \rho(\mathbf{r})$ $\approx \frac{\rho_{\Delta \lambda}(\mathbf{r})-\rho_{\mathrm{R}}(\mathbf{r})}{\Delta \lambda}$. In practice, instead of starting the SCF for the perturbed system from atom based initial guesses, we restart with $\rho_{\mathrm{R}}(\mathbf{r})$ resulting in convergence within few SCF steps.

\section{Higher order derivatives}

Møller-Plesset (MP) perturbation theory ${ }^{48,49}$ is used to estimate correlation energy corrections based on converged Hartree-Fock results. The derivation of higher order corrections in MP theory is equivalent to the $m$ th order alchemical derivative. Here, instead of the two-particle operator for electron-electron interaction as perturbation in MP theory, the alchemical perturbation operator $H_{T}-H_{R}$ can be used. Within IPA, the MP formula can be directly applied to obtain any $m$ th order derivative.

\section{E. Predicting changes in covalent bonds}

For the study of covalent bonds, we focus on the changes in binding potential due to alchemical coupling. We consider the difference in total potential energy between two covalently bound fragments at two arbitrary interatomic distances $d$ and $d_{0}$,

$$
\Delta E\left(d, d_{0}\right)=E(d)-E\left(d_{0}\right) .
$$

If, for example, $d_{0}$ is large and $d$ is the geometry minimum, $\Delta E$ becomes the bond dissociation energy. We are interested in changes of $\Delta E\left(d, d_{0}\right)$ as a function of $d$ due to alchemical changes for a fixed $d_{0}$. More specifically, we couple a reference to target system via the corresponding Hamiltonians yielding expectation values as a function of $\lambda$,

$$
\begin{aligned}
\Delta E_{\lambda}\left(d, d_{0}\right)= & E_{\lambda}(d)-E_{\lambda}\left(d_{0}\right) \\
= & \left\langle H_{R}(d)+\lambda\left(H_{T}(d)-H_{R}(d)\right)\right\rangle \\
& -\left\langle H_{R}\left(d_{0}\right)+\lambda\left(H_{T}\left(d_{0}\right)-H_{R}\left(d_{0}\right)\right)\right\rangle .
\end{aligned}
$$

As $\lambda$ goes from 0 to 1 , the two components in Eq. (9) change from reference $\left(E_{\mathrm{R}}(d), E_{\mathrm{R}}\left(d_{0}\right)\right)$ to target $\left(E_{\mathrm{T}}(d), E_{\mathrm{T}}\left(d_{0}\right)\right)$ compound. The truncated Taylor expansion based estimate of the target compound's potential is then obtained via

$$
\begin{aligned}
\Delta E_{\mathrm{T}}\left(d, d_{0}\right) & \approx \Delta E_{\mathrm{T}}^{(m)}\left(d, d_{0}\right) \\
& =\Delta E_{\mathrm{R}}\left(d, d_{0}\right)+\sum_{k=1}^{m} \frac{1}{k !} \partial_{\lambda}^{k} \Delta E_{\lambda}\left(d, d_{0}\right),
\end{aligned}
$$

where the superscript $m$ stands for Taylor expansion with $m$ terms, as a function of bond-length $d$ for vertical alchemical changes. Since $\Delta E_{\mathrm{T}}$ is the property of interest, the subscript $\mathrm{T}$, $\lambda$, and the dependency of $d_{0}$ will be omitted for the rest of this work, unless otherwise noted. In this study, we investigated orders up to $m=4$ for the stretching of $\mathrm{H}_{2}^{+}$, and up to $m=2$ for all other molecules. For a fixed $d_{0}$, the first order estimate is calculated according to

$$
\begin{aligned}
\Delta E_{\mathrm{T}}^{(1)}(d)= & \left(E_{\mathrm{R}}(d)+\left.\partial_{\lambda} E_{\lambda}(d)\right|_{\lambda=0}\right) \\
& -\left(E_{\mathrm{R}}\left(d_{0}\right)+\left.\partial_{\lambda} E_{\lambda}\left(d_{0}\right)\right|_{\lambda=0}\right) \\
= & E_{\mathrm{R}}(d)+\int d \mathbf{r} \rho_{\mathrm{R}}(d, \mathbf{r})\left[v_{\mathrm{T}}(d, \mathbf{r})-v_{\mathrm{R}}(d, \mathbf{r})\right] \\
& +\left[V_{\mathrm{T}}(d)-V_{\mathrm{R}}(d)\right]-E_{\mathrm{R}}\left(d_{0}\right) \\
& -\int d \mathbf{r} \rho_{\mathrm{R}}\left(d_{0}, \mathbf{r}\right)\left[v_{\mathrm{T}}\left(d_{0}, \mathbf{r}\right)-v_{\mathrm{R}}\left(d_{0}, \mathbf{r}\right)\right] \\
& -\left[V_{\mathrm{T}}\left(d_{0}\right)-V_{\mathrm{R}}\left(d_{0}\right)\right],
\end{aligned}
$$

and the second order estimate correspondingly,

$$
\begin{aligned}
\Delta E_{\mathrm{T}}^{(2)}(d)= & \left(E_{\mathrm{R}}(d)+\left.\partial_{\lambda} E_{\lambda}(d)\right|_{\lambda=0}+\left.\frac{1}{2} \partial_{\lambda}^{2} E_{\lambda}(d)\right|_{\lambda=0}\right) \\
& -\left(E_{\mathrm{R}}\left(d_{0}\right)+\left.\partial_{\lambda} E_{\lambda}\left(d_{0}\right)\right|_{\lambda=0}+\left.\frac{1}{2} \partial_{\lambda}^{2} E_{\lambda}\left(d_{0}\right)\right|_{\lambda=0}\right),
\end{aligned}
$$

using above discussed expressions for the CP, IPA, and FD definitions of the second order derivative.

Since $d$ and $d_{0}$ in Eq. (9) are arbitrary, one can obtain the binding curve by scanning $d$ for any fixed $d_{0}$. The predictive power, however, happens to depend on $d_{0}$. For this reason, we optimize $d_{0}$ such that the integrated error in dissociation region is minimal. As shown in Fig. 3 and discussed below, an empirical linear relationship exists between equilibrium bond 
length of target molecule $d_{\mathrm{eq}}^{\mathrm{T}}$ and $d_{\mathrm{opt}}$,

$$
d_{\mathrm{opt}} \approx 0.76 d_{\mathrm{eq}}^{\mathrm{T}}+0.97 \AA .
$$

$d_{0}$ is determined according to Eq. (14) for all vertical changes. If $d_{\mathrm{eq}}^{\mathrm{T}}$ is not known, it can easily be estimated with semiempirical quantum chemistry methods.

For non-vertical changes, we use the equilibrium distance $d_{\mathrm{eq}}$ in the reference molecule as starting reference geometry, and we also fix $d_{0}$ to the same value, resulting in $\Delta E^{(m)}\left(d_{\mathrm{eq}}\right)$ $=0$. Eq. (12) simplifies in this case,

$$
\begin{aligned}
\Delta E^{(1)}(d)= & \int d \mathbf{r} \rho_{\mathrm{R}}\left(d_{\mathrm{eq}}, \mathbf{r}\right)\left[v_{\mathrm{T}}(d, \mathbf{r})-v_{\mathrm{T}}\left(d_{\mathrm{eq}}, \mathbf{r}\right)\right] \\
& +\left[V_{\mathrm{T}}(d)-V_{\mathrm{T}}\left(d_{\mathrm{eq}}\right)\right],
\end{aligned}
$$

and so does Eq. (13) for second order estimates.

\section{F. Error measures}

For bond lengths, we quantify the predictive power of the Taylor expansions by evaluating the deviation of prediction from the DFT bond length $\Delta d_{\mathrm{eq}}=d_{\mathrm{eq}}^{(m)}-d_{\mathrm{eq}}$, where $d_{\mathrm{eq}}^{(m)}$ stands for the predicted equilibrium distance of $\Delta E^{(m)}$. We calculate the deviation of the predicted energy at $d_{\mathrm{eq}}^{(m)}$ from the DFT energy at the DFT minimum, $\Delta E_{\mathrm{eq}}=\Delta E^{(m)}\left(d_{\mathrm{eq}}^{(m)}\right)-\Delta E\left(d_{\mathrm{eq}}\right)$. The deviation in harmonic vibration frequency, $\Delta \omega=\omega^{(m)}-\omega$, of the bond stretching is also included in order to quantify the accuracy of the stiffness of the predicted binding potential. The vibration frequency is computed from the curvature of cubic spline interpolated binding potential, $\omega=\frac{1}{2 \pi} \sqrt{\frac{k_{\mathrm{eq}}}{\mu}}$, where $k_{\mathrm{eq}}=\partial_{d}^{2} \Delta E\left(d_{\mathrm{eq}}\right)$ and $\mu$ is the reduced mass. Finally, we measure the integrated mean absolute error (MAE) for the dissociative tail, defined as

$$
\mathrm{MAE}=\frac{1}{\left|d_{\max }-d_{\mathrm{eq}}^{(m)}\right|} \int_{d_{\mathrm{eq}}^{(m)}}^{d_{\max }} d x\left|\Delta E^{(m)}(x)-\Delta E(x)\right|,
$$

for vertical iso-valence-electronic changes. Note that while in principle one would like $d_{\max } \rightarrow \infty, d_{\max }$ has been set to correspond roughly to the inflection point, due to the issues of a single determinant method, such as DFT, for describing covalent bond-dissociation. This shortcoming is also evident from comparison of DFT to coupled-cluster single doubles perturbative triples $(\mathrm{CCSD}(\mathrm{T}))$ curves shown in Fig. 3. Note that this aspect is irrelevant for alchemical predictions: If a more reliable reference method had been used, the error integration could have easily been expanded to include the entire dissociative tail. These four quantities provide a numerical indication of how good a prediction is. For a perfect prediction, one would expect $\left(\Delta E_{\mathrm{eq}}, \Delta d_{\mathrm{eq}}, \Delta \omega, \mathrm{MAE}\right)=(0,0,0,0)$. Note that we compare the predictions to DFT. This is an arbitrary choice, any other QM method could have been applied just as well.

\section{G. Computational details}

Alchemical interpolations of molecules containing elements from different rows in the periodic table can still be iso-electronic if effective core or pseudopotentials (PPs) are used, resulting in a constant number of valence electrons. ${ }^{12}$ For example, one can couple carbon to silicon using just four valence electrons. Non-local PPs are widely used to mimic the presence of core electrons in atoms ${ }^{50}$ and are amenable to the tuning of a wide range of properties including dispersion forces, band-gap, or vibrational frequencies. ${ }^{51-53}$ The non-local external potential $v_{\lambda}(\mathbf{r})$ in Eq. (3) then becomes

$$
v_{\lambda}\left(\mathbf{r}, \mathbf{r}^{\prime}\right)=\sum_{I}^{N_{I}}\left((1-\lambda) v_{I}^{\mathrm{R}}\left(\mathbf{r}, \mathbf{r}^{\prime}\right)+\lambda v_{I}^{\mathrm{T}}\left(\mathbf{r}, \mathbf{r}^{\prime}\right)\right)
$$

where $v_{I}^{\mathrm{R}}$ and $v_{I}^{\mathrm{T}}$ are PPs for $Z_{I}^{\mathrm{R}}$ and $Z_{I}^{\mathrm{T}}$, respectively. Note that $v_{\lambda}\left(\mathbf{r}, \mathbf{r}^{\prime}\right)$ in Eq. (17) and $v_{\lambda}(\mathbf{r})$ in Eq. (3) result in different coupling Hamiltonians, and therefore different $\lambda$-dependencies of the energy and its derivatives.

All results have been obtained within the BornOppenheimer approximation, where nuclei are clamped; nuclear repulsion $V_{\lambda}$ is decoupled from the electronic wavefunction and is added as a geometry- and $\lambda$-dependent constant to the electronic energy. Nuclear-nuclear repulsion energy is computed automatically by most QM codes. However, it must be removed and recomputed independently for $V_{\lambda}$ according to Eq. (3) to avoid self-repulsion between transmutating atoms. Throughout the present study, standard atomic and plane-wave basis functions, linearly interpolated PPs, as well as the PBE xc potential ${ }^{54}$ within Kohn-Sham DFT are used. The scanning of $0.5 \AA \leq d \leq 3.0 \AA$ is carried out with increments $\Delta d=0.1 \AA$. For each prediction order $m, \Delta E^{(m)}(d)$ are interpolated with cubic splines, from which the stiffness $\partial_{d}^{2} \Delta E^{(m)}\left(d_{\mathrm{eq}}^{(m)}\right)=k_{\mathrm{eq}}$ is computed. All density volumetric data are printed into Gaussian CUBE files, from which integrated density slices are calculated.

\section{Details for vertical iso-valence-electronic changes}

Numerical results for vertical iso-valence-electronic alchemical changes (discussed in Secs. III A and III B) have been obtained with CPMD, ${ }^{55}$ a plane wave basis with 100 Ry cutoff, and Goedecker PPs. ${ }^{56-58}$ The periodic supercell size is $20 \times 15 \times 15 \AA^{3}$, and one heavy atom is fixed at (7.5 $\AA$, 7.5 $\AA$, 7.5 $\AA$ ) while the stretching atom shifts along $+x$-axis. For each geometry, heavy atoms are mutated to other elements in the same column of the periodic table while all H's are fixed at the same location as in the reference compound.

Since Eq. (17) is a non-local operator, Eqs. (4) and (5) need to be converted to wavefunction expressions. The first order derivative for the Hamiltonian $H_{\mathrm{R} \rightarrow \mathrm{T}}$ is evaluated using RESTART files in which the reference compound's density and wavefunctions have been stored: $\partial_{\lambda} E=\left\langle\partial_{\lambda} H\right\rangle_{\mathrm{R}}$ $=E_{\mathrm{T}}\left[\rho_{\mathrm{R}}\right]-E_{\mathrm{R}}\left[\rho_{\mathrm{R}}\right]$. And the second order derivative is evaluated correspondingly relying on FD, $\partial_{\lambda}^{2} E \approx \frac{\left\langle\partial_{\lambda} H\right\rangle_{\Delta \lambda}-\left\langle\partial_{\lambda} H\right\rangle_{\mathrm{R}}}{\Delta \lambda}$, with $\Delta \lambda=0.05$. Wavefunctions of reference compound are used for $\Delta E^{(1)}$, while $\Delta E_{\mathrm{FD}}^{(2)}$ is evaluated by FD with linearly interpolated $\mathrm{PP}$ parameters.

CCSD(T) results obtained for $\mathrm{HCl}$ and $\mathrm{HBr}$ have been computed using Gaussian09 ${ }^{59}$ in aug-cc-pVTZ ${ }^{60}$ basis and default input parameters. 


\section{Details for non-vertical iso-electronic changes}

Numerical results for non-vertical iso-electronic alchemical changes have been obtained using atom centered basissets. Restricted open-shell Hartree-Fock calculations have been carried out using Cartesian aug-cc-pVTZ basis set ${ }^{60}$ for $\mathrm{H}_{2}^{+}$(discussed in Sec. III D 1). Eq. (6) and higher order derivatives are evaluated analytically by Gaussian expansion of MOs. Reference geometry is first relaxed by Gaussian $09^{59}$ and the converged MO coefficients are extracted to evaluate orbital integrals. NWChem ${ }^{61}$ is used to scan $\Delta E$ as a function of $\lambda$ in Fig. 5(d) along alchemical path with discretization $\Delta \lambda=0.01$. It is done by reassigning nuclear charges in the system.

Non-vertical alchemical changes in 10-electron molecules (discussed in Sec. III D 2) have been calculated using the uncontracted Cartesian Def2TZVP basis set. ${ }^{62}$ Uncontracted neon basis is used for all second row atoms. Additional hydrogen basis functions are placed along the stretching pathway, from $d=0.5 \AA$ to $d=3.0 \AA$ in increments $\Delta d=0.1 \AA$ A. All systems with integer nuclear charges have been calculated using Gaussian $09^{59}$ while systems with fractional nuclear charges have been calculated using NWChem ${ }^{61}$ with discretization $\Delta \lambda=0.01$. For each $0 \leq \lambda \leq 1$, the atomic density for SCF initial guess iterates through $\{\mathrm{C}, \mathrm{N}$, $\mathrm{O}, \mathrm{F}, \mathrm{Ne}\}$ to ensure convergence. In all Gaussi an and NWChem calculations, we used Cartesian/real spherical harmonic basis functions.

\section{RESULTS AND DISCUSSIONS}

\section{A. Vertical iso-valence-electronic changes of $\mathrm{X}-\mathrm{H}$}

\section{Predicted potentials}

Using Taylor expansions binding potentials have been estimated for covalent bonds involving hydrogen $(\mathrm{X}-\mathrm{H})$ for the following 12 molecules with 8 valence electrons: $\mathrm{CH}_{4}, \mathrm{NH}_{3}, \mathrm{H}_{2} \mathrm{O}, \mathrm{HF}$ (second period); $\mathrm{SiH}_{4}, \mathrm{PH}_{3}, \mathrm{H}_{2} \mathrm{~S}$, $\mathrm{HCl}$ (third period); and $\mathrm{GeH}_{4}, \mathrm{AsH}_{3}, \mathrm{H}_{2} \mathrm{Se}, \mathrm{HBr}$ (fourth period). Numerical results for vertical first (red) and second (blue) order truncated Taylor series estimates, calculated with Eqs. (12) and (13), feature in Fig. 1. They measure the change in $\mathrm{X}-\mathrm{H}$ binding energy as one goes from reference to target compound.

We first note that the entire potential is reproduced in semi-quantitative fashion for all combinations of reference/target molecules. The precise predictive power strongly depends on the choice of reference/target molecule pair, on the choice of $d_{0}$, and on the expansion going up to first or second order. First order estimates among molecules with elements from the third or fourth row are very accurate (see Fig. 1, bottom and mid-row in mid- and bottom panels, respectively). By contrast, predicting, or starting with, second row elements consistently yields worse results. Inclusion of second order corrections does not necessarily lead to improved performance. Second order truncated Taylor series estimates only yield more accurate predictions than first order when the reference molecule contains heavier elements than the target molecule. For example, if we predict HF using
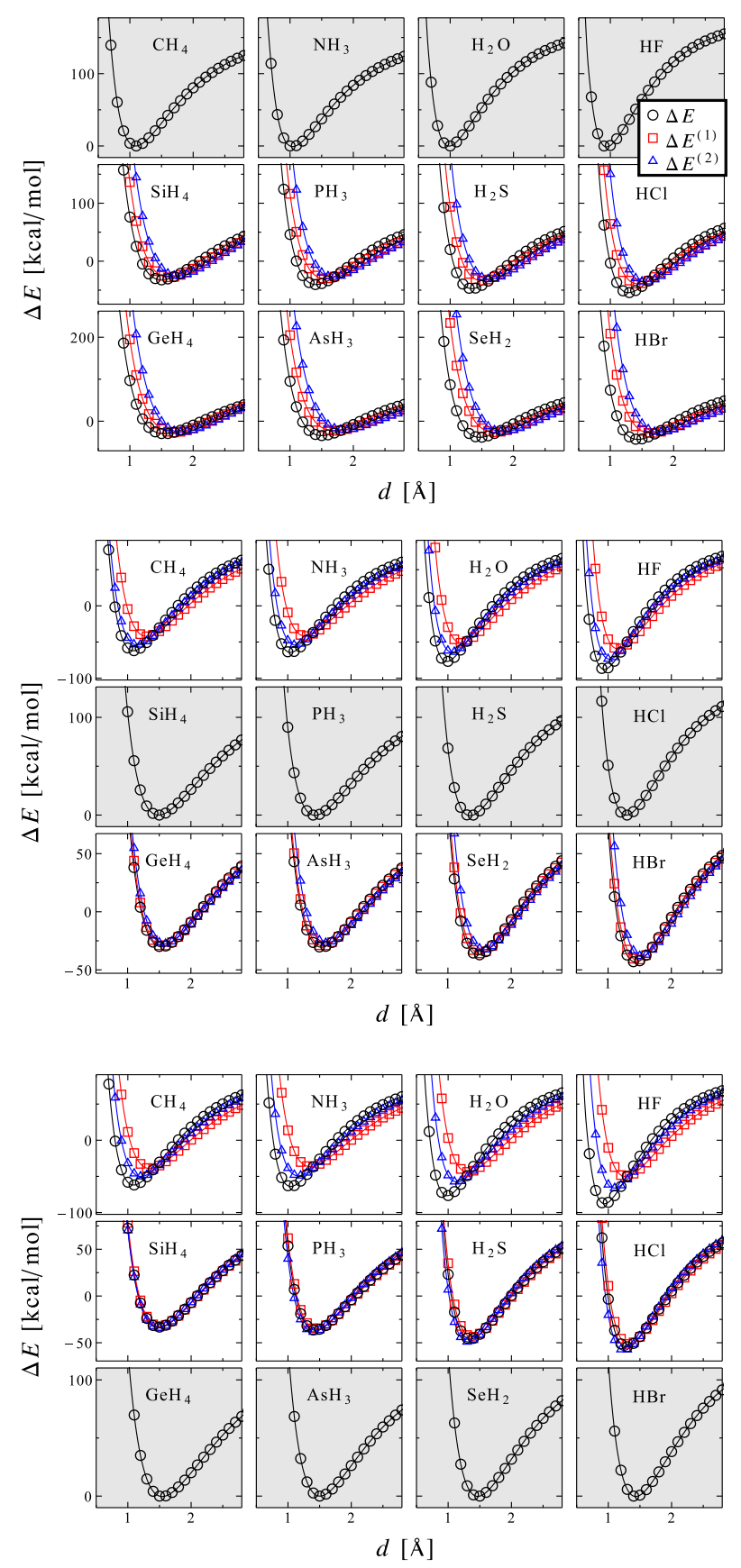

FIG. 1. $\Delta E$ is shown as a function of $d$ in Eq. (11). White background panels: true (black circles), first (red squares), and second (blue triangles) order predictions of changes in the covalent bond potential of hydrogen due to vertical alchemical interpolations. Gray background panels: the true potentials of the reference compounds employed for first and second order predictions.

$\mathrm{HBr}$ as a reference, the second order prediction is more accurate than first order. For the inverse prediction (i.e., $\mathrm{HBr}$ from HF), however, first order is more accurate than second order.

The performance of truncated Taylor series dramatically varies depending on the choice of the $d_{0}$ value. The top panel in Fig. 2 illustrates this for $\Delta E\left(2 \AA, d_{0}\right)$ for $\mathrm{HF} \rightarrow \mathrm{HBr}$ as a function of $\lambda$, once with $d_{0}=0.94 \AA=d_{\mathrm{eq}}$ the equilibrium bond length of HF- and once with $d_{0}=1.57 \AA=d_{\text {opt }}$, a value for $d_{0}$ which happens to linearize $\Delta E$ in $\lambda$. While the coupling 

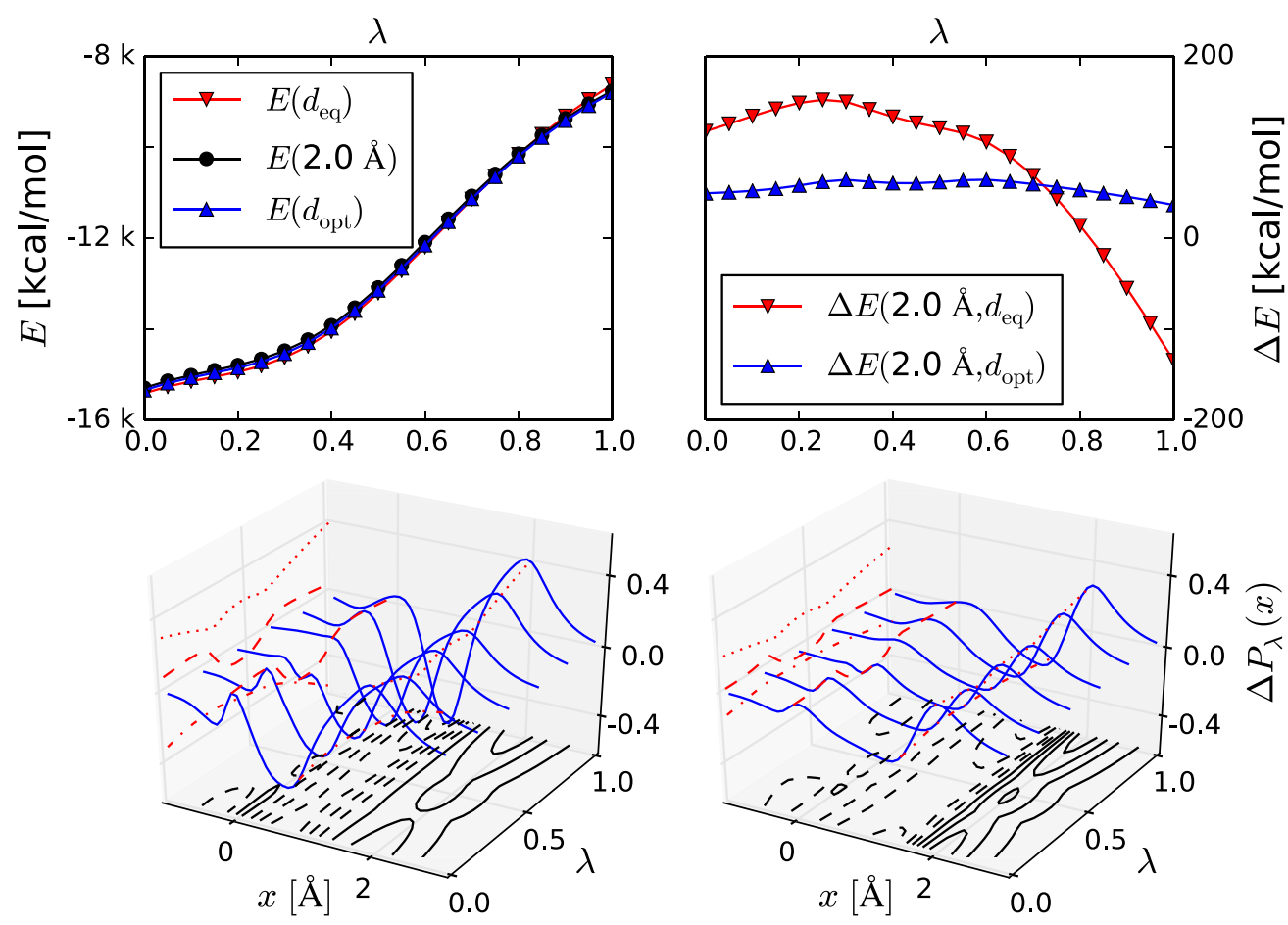

FIG. 2. Alchemical coupling of $\operatorname{HF}(\lambda=0)$ to $\operatorname{HBr}(\lambda=1)$. TOP panel: $E$ (LEFT) and $\Delta E$ (RIGHT) where $d_{\mathrm{eq}}=0.94 \AA$ (red) denotes the equilibrium bond length of reference molecule HF, and $d_{\mathrm{opt}}=1.57 \AA$ (blue) linearizes $\Delta E$. BOTTOM panel: integrated valence electron density difference slices between H-X at $d=2 \AA$ and at $d_{\mathrm{opt}}$ (RIGHT) and $d_{\mathrm{eq}}$ (LEFT), respectively, $\Delta P_{\lambda}(x)=\int d y d z\left[\rho_{\lambda}(\mathbf{r}, d)-\rho_{\lambda}\left(\mathbf{r}, d_{0}\right)\right]$. Dependence on $\lambda$ is shown for the same vertical interpolations, left and right corresponding to the non-linear (red) and linearized (blue) $\Delta E$ curves in right-hand TOP panel. Density changes at heavy atom and hydrogen positions are highlighted as red dashed and dotted/dashed-dotted lines, respectively.

path of total energies is hardly distinguishable for $E(2 \AA)$, $E(1.57 \AA)$, and $E(0.94 \AA), \Delta E$ is strongly dependent on the choice of $d_{0}$. By choosing $d_{0}=1.57 \AA, \Delta E(2 \AA, 1.57 \AA)$ in Eq. (10) becomes nearly linear, while plotting $\Delta E(2 \AA, 0.94 \AA)$ reveals substantial curving. This is why, when choosing the right $d_{0}$, first order predictions of $\Delta E$ can be very predictive.

The top panel in Fig. 2 also explains why second order estimates can be worse than first order, and why this changes for the reverse coupling: On the side of the lighter element
( $\lambda \rightarrow 0$ ), a weak convexity is noticeable in $\Delta E$ (blue line), despite the overall concavity of the path. The presence of inflection points will always lead to a deterioration of second order predictions, implying a more accurate first order estimate. On the other side $(\lambda \rightarrow 1)$, no such inflection point exists and the second order term results in the expected improvement of the prediction. For the other compound pairs shown in Fig. 1, similar observations can be made for the attractive part of the bonding potential (see also $\Delta E\left(2 \AA, d_{0}\right)$
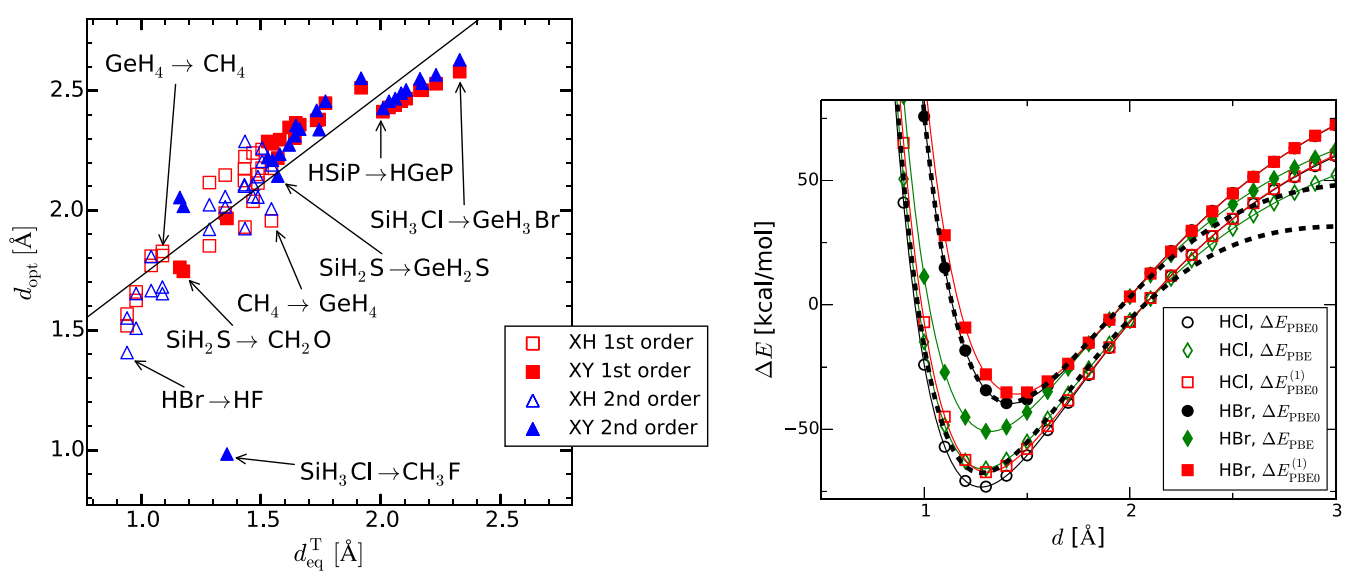

FIG. 3. LEFT: Scatter plot of optimized reference bond length $d_{\mathrm{opt}}$ versus equilibrium bond length of target molecule, denoted by $d_{\mathrm{eq}}^{\mathrm{T}}$. Linear regression gives $d_{\mathrm{opt}}=0.76 d_{\mathrm{eq}}+0.97 \AA$ with MAE $=0.11 \AA$ and RMSE $=0.15 \AA$. First order (red empty squares) and second order (blue empty triangles) $d_{\mathrm{opt}}$ of covalent X-H bond stretching, as well as first order (red filled squares) and second order (blue filled triangles) $d_{\mathrm{opt}}$ of $\mathrm{X}-\mathrm{Y}, \mathrm{X}=\mathrm{Y}, \mathrm{X} \# \mathrm{Y}$ stretching are shown, where -, =, \# stand for single, double, and triple bonds. Some of the alchemical paths are highlighted by black arrows. All numbers are given in Tables SI and SII. ${ }^{72}$ RIGHT: Alchemical predictions can be more accurate than approximated density functionals. Covalent binding potentials obtained from alchemical PBE0 estimate (red squares) and ordinary PBE (green diamonds) for $\mathrm{HBr}$ (full symbols) and $\mathrm{HCl}$ (empty symbols). The alchemical estimate corresponds to Eq. (12) using PBE0 density of $\mathrm{HBr}(\mathrm{HCl})$ in order to predict $\mathrm{HCl}(\mathrm{HBr})$. For comparison, corresponding $\mathrm{CCSD}(\mathrm{T})$ results are shown as well (dashed). 
curves in supplementary material ${ }^{72}$ ). We have found that the inflection point near $\lambda=0$ occurs only when the reference molecule has the lighter element. Conversely, no inflection point has been observed for atoms transmutating upward the column. We believe that this behaviour is due to the specifics of the employed PPs. Future studies will show why this is the case, and if similar trends hold for other PPs.

\section{Integrated error}

Prediction errors for energy minima, equilibrium bond lengths, force constants, and integrated error in dissociation region (calculated as described in Sec. II F) have been obtained for all predictions in Fig. 1 and are reported in Table SI. ${ }^{72}$ The results lend quantitative support for the observations articulated above. In particular, these results suggest that chemical accuracy can be obtained when using first order Taylor series based estimates among compounds containing third and fourth row elements. Second order based predictions are always worse except when a molecule with heavier element is used as a reference to predict a molecule with lighter one, for example, $\mathrm{HBr} \rightarrow \mathrm{HF}$.

The best prediction performance is found for first order based estimates using reference molecules containing third row elements $\left(n_{\mathrm{R}}=3\right)$ in order to predict target molecules made up of fourth row elements $\left(n_{\mathrm{T}}=4\right)$. The overall average deviation from reference bonding potential energies and integrated error is $\sim 2.5 \mathrm{kcal} / \mathrm{mol}$. Corresponding predictions of equilibrium distances deviate at most by $0.03 \AA$, and the vibration frequencies deviate not more than $32 \mathrm{~cm}^{-1}$. Second order estimates for the same third and fourth row combinations give slightly worse results. The worst predictions are found if the coupled molecules skip a row, i.e., involve elements from second and fourth row-for first as well as second order truncated Taylor expansions. This is not surprising as the central atom's electron density must accommodate the most severe contractions/expansions for such interpolations. Moreover, second order overcorrections can also be found (Table SI) ${ }^{72}$ whenever molecules containing fourth row elements are used to predict molecules containing third row elements: both, predicted energy minimum and equilibrium bond length, show negative deviations.

\section{Alchemical predictions do not commute}

We note the asymmetry in the predictive power of first order based predictions which is due to the lack of commutation: In general $\left.\partial_{\lambda} E\right|_{\lambda=0} \neq-\left.\partial_{\lambda} E\right|_{\lambda=1}$, except if reference and target Hamiltonian happen to differ only by translation, rotation, or parity (enantiomers, i.e., without accounting for parity violation). Within our restricted case of linear interpolations of iso-electronic systems, the perturbing potential does differ only by sign. The integral over its product with the electron density, however, differs in general, i.e., $\left\langle H_{\mathrm{A}}-H_{\mathrm{B}}\right\rangle_{\mathrm{B}}=\int d \mathbf{r} \rho_{\mathrm{B}}\left(v_{\mathrm{A}}-v_{\mathrm{B}}\right) \neq \int d \mathbf{r} \rho_{\mathrm{A}}\left(v_{\mathrm{A}}\right.$ $\left.-v_{\mathrm{B}}\right)=-\left\langle H_{\mathrm{B}}-H_{\mathrm{A}}\right\rangle_{\mathrm{A}}$. As such, the error in estimating A based on $\mathrm{B}$ will not be the same as the error in estimating $\mathrm{B}$ based on $\mathrm{A}$. Results in Table $\mathrm{SI}^{72}$ suggest that predictions downward the columns in the periodic table are more accurate than upward. For example, predicting $\mathrm{HBr}$ using $\mathrm{HF}$ as a reference, a better estimate is obtained (error $=+25.7 \mathrm{kcal} / \mathrm{mol}$ ) than for predicting $\mathrm{HF}$ using $\mathrm{HBr}$ as a reference (error $=+61.1 \mathrm{kcal} / \mathrm{mol}$ ). Correspondingly, predicting $\mathrm{HCl}$ using $\mathrm{HBr}$ has an error $=+5.4 \mathrm{kcal} / \mathrm{mol}$, while the prediction of $\mathrm{HBr}$ using $\mathrm{HCl}$ has only an error of $+3.6 \mathrm{kcal} / \mathrm{mol}$. Similar observations hold for bond lengths and force constants. The asymmetry is also illustrated in Fig. 2. $\Delta E\left(d, d_{0}\right)$ is not necessarily symmetric with respect to $\lambda=0.5$ for a given choice of $\left(d, d_{0}\right)$. Consequently, truncated Taylor series based predictions from either ends will not be equally accurate.

\section{Chemical accuracy}

We have seen that very accurate, yet inexpensive, first order alchemical estimates can be made for vertical alchemical changes between third and fourth row elements according to Eq. (4) —once the density is converged for a given reference molecule. Then, an interesting question is if the alchemical accuracy is on the same order of magnitude as common approximations made when solving Schrödinger's equation. We have investigated this point for alchemical coupling of $\mathrm{HBr}$ and $\mathrm{HCl}$ using hybrid and generalized gradient approximated DFT. When using PBE0 ${ }^{63}$ as the method for the reference compound, we find the first order based alchemical predictions according to Eq. (12) to be in better agreement with the PBE0 results for the target compound than true generalized gradient based approximation PBE. ${ }^{64} \mathrm{Fig} .3$ illustrates this point for the covalent binding potentials of $\mathrm{HCl}$ and $\mathrm{HBr}$ calculated using PBE0, PBE0 based vertical first order alchemical predictions, and PBE. For all interatomic distances in the dissociative tail, the alchemical prediction (squares) is closer to PBE0 (circles) than PBE (diamonds). For the repulsive part of the potential, the alchemical prediction is substantially better than PBE for $\mathrm{HBr}$ and slightly worse than PBE for $\mathrm{HCl}$. For comparison, we also included $\operatorname{CCSD}(\mathrm{T})$ results. These results amount to numerical evidence that the predictive power of vertical alchemical predictions can exceed the accuracy of common DFT approximations for third or fourth row elements-if a sufficiently accurate electron density is provided for the reference compound.

\section{B. Vertical iso-valence-electronic changes involving single, double, and triple bonds}

\section{Predicted potentials}

Having discussed covalent bonds involving hydrogen, we now turn to single $\left(\mathrm{XH}_{3}-\mathrm{Y}\right)$, double $\left(\mathrm{XH}_{2}=\mathrm{Y}\right)$, and triple $(\mathrm{HX \# Y)}$ bonds among $p$-block elements. Since third row elements can either be alchemically compressed to the corresponding second row $(n=2)$ element in the same column, or expanded to the fourth row $(n=4)$ element, we chose third row $(n=3)$ based reference systems for single, double, and triple bonds, namely, $\mathrm{SiH}_{3} \mathrm{Cl}, \mathrm{SiH}_{2} \mathrm{~S}$, and $\mathrm{HSiP}$. The resulting eight alchemical paths are combinations of changing the $\mathrm{Si}$ atom $(\mathrm{Si} \rightarrow \mathrm{C}, \mathrm{Si} \rightarrow \mathrm{Ge})$ or its binding partner $(\mathrm{Cl} \rightarrow \mathrm{F}, \mathrm{Cl} \rightarrow \mathrm{Br}, \mathrm{S} \rightarrow \mathrm{O}, \mathrm{S} \rightarrow \mathrm{Se}, \mathrm{P} \rightarrow \mathrm{N}, \mathrm{P} \rightarrow \mathrm{As})$. 
In Fig. 4 first and second order alchemical predictions, again calculated with Eqs. (12) and (13), are shown for the bonding potential using vertical transmutations from the three reference molecules.

More specifically, single bond predictions have been investigated for making predictions using $\mathrm{SiH}_{3} \mathrm{Cl}$ as a
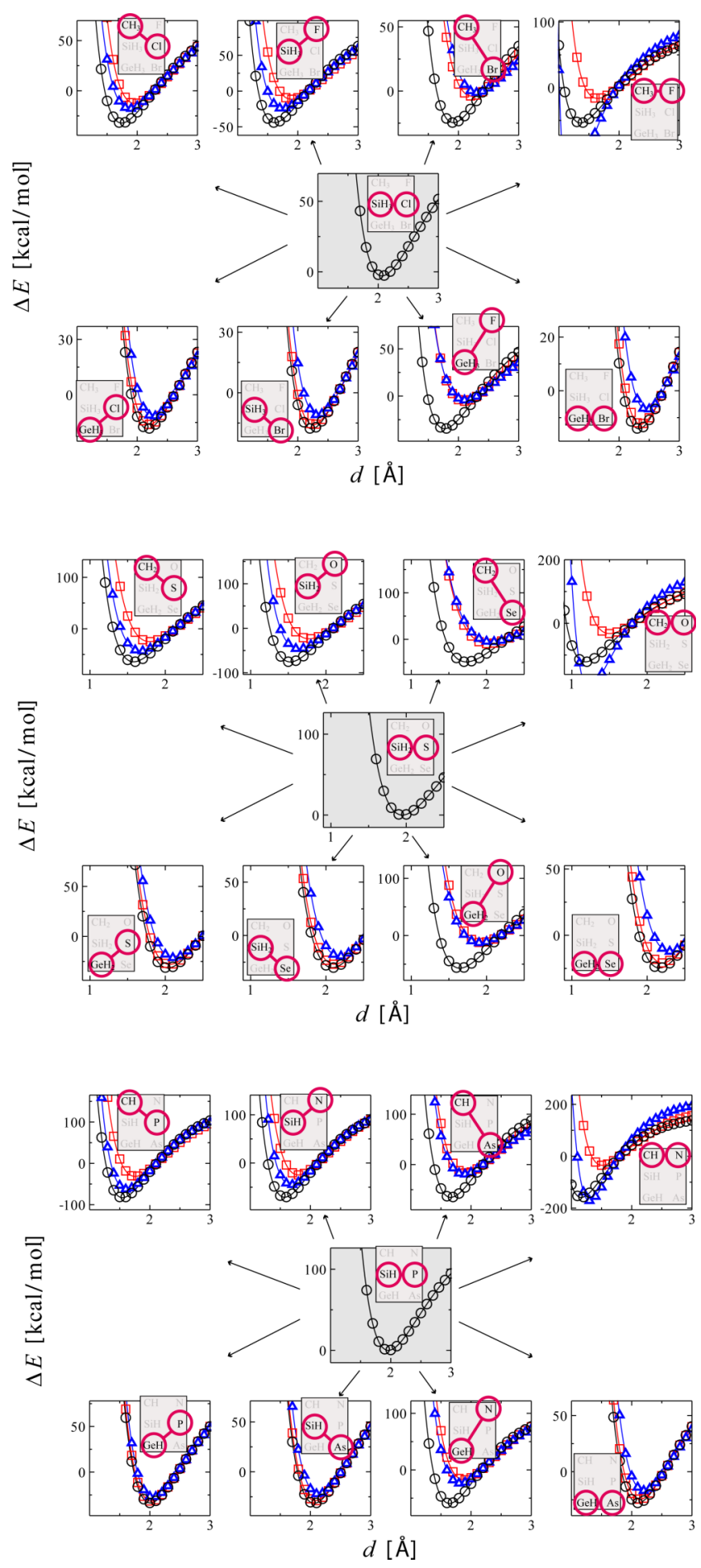

FIG. 4. Alchemical predictions of single (top), double (middle), and triple (bottom) bond potentials. Curves are shown for eight target systems (specified as insets), iso-electronic with reference (gray background) molecule $\mathrm{SiH}_{3} \mathrm{Cl}$ (upper panel), $\mathrm{SiH}_{2} \mathrm{~S}$ (middle panel), and $\mathrm{HSiP}$ (bottom panel). Potentials correspond to true (black circles), first (red squares), and second (blue triangles) order vertical alchemical predictions of heavy atom bond dissociation curves. reference compound for the eight following molecules with 14 valence electrons: $\mathrm{CH}_{3} \mathrm{~F}, \mathrm{CH}_{3} \mathrm{Cl}, \mathrm{CH}_{3} \mathrm{Br}\left(n_{\mathrm{X}}=2\right)$; $\mathrm{SiH}_{3} \mathrm{~F}$, $\mathrm{SiH}_{3} \mathrm{Br}\left(n_{\mathrm{X}}=3\right)$; and $\mathrm{GeH}_{3} \mathrm{~F}, \mathrm{GeH}_{3} \mathrm{Cl}$, and $\mathrm{GeH}_{3} \mathrm{Br}\left(n_{\mathrm{X}}=4\right)$. For double bonds, we have considered predictions for the following eight unsaturated molecules 12 valence electrons and using $\mathrm{SiH}_{2} \mathrm{~S}$ as a reference compound: $\mathrm{CH}_{2} \mathrm{O}, \mathrm{CH}_{2} \mathrm{~S}$, $\mathrm{CH}_{2} \mathrm{Se}\left(n_{\mathrm{X}}=2\right) ; \mathrm{SiH}_{2} \mathrm{O}, \mathrm{SiH}_{2} \mathrm{Se}\left(n_{\mathrm{X}}=3\right) ;$ and $\mathrm{GeH}_{2} \mathrm{O}$, $\mathrm{GeH}_{2} \mathrm{~S}$, and $\mathrm{GeH}_{2} \mathrm{Se}\left(n_{\mathrm{X}}=4\right)$. And finally for triple bonds, we have studied the following eight molecules with 10 valence electrons and using $\mathrm{HSiP}$ as a reference compound: $\mathrm{HCN}$, HCP, HCAs $\left(n_{\mathrm{X}}=2\right)$; HSiN, HSiAs $\left(n_{\mathrm{X}}=3\right)$; and HGeN, HGeP, and HGeAs $\left(n_{\mathrm{X}}=4\right)$.

Numerical results in Fig. 4 indicate qualitatively correct behavior for all predictions. Regarding quantitative performance, the accuracy of the alchemical prediction of $\Delta E\left(d, d_{0}\right)$ exhibits similar trends as the one discussed above in the case of vertical changes in the hydrogen containing single bond: First order predictions (red) systematically achieve strong predictive power whenever the change involves the coupling of the third row element to a fourth row element. Corresponding second order predictions (blue) deteriorate the accuracy due to inflection points near $\lambda=0$. If the coupling involves one lighter element from the second row, the prediction is no longer quantitative. However, in these cases, second order predictions provide a slightly superior prediction. If both atoms are simultaneously transmutated to lighter atoms from the second row, e.g., $\mathrm{SiH}_{3} \mathrm{Cl} \rightarrow \mathrm{CH}_{3} \mathrm{~F}$, second order estimates over-correct (change of sign) the first order prediction. In the case of one element transmutating upward the column, the other downward, the second order estimate is hardly distinguishable from the first order estimate. We believe that the reason for this is that the coupling to the lighter element on the one site in the molecule yields the concave behavior leading to an improvement in the prediction, while the coupling to the heavier element on the other site in the molecule yields the convex behavior with the inflection point, leading to a deterioration of the prediction. Effectively, these two effects cancel each other and result in the same predictive accuracy as the one obtained for the first order estimate. This rationalization rests on the assumption that the discussion of Fig. 2 can also be applied to a linear combination of effects at different transmutating sites.

\section{Integrated errors}

Above observations are consistent with the quantitative integrated prediction error measures (definitions in Sec. II F) summarized in Table SII. ${ }^{72}$ All first order based predictions of target molecules implying a transmutation downward the periodic table (columns 4/3, 3/4, 4/4) exhibit chemical accuracy with at most $1.83 \mathrm{kcal} / \mathrm{mol}$ deviation in minimal energy $\left(\mathrm{GeH}_{2} \mathrm{~S}\right)$, at most $0.04 \AA$ deviation in bond length $\left(\mathrm{GeH}_{2} \mathrm{Se}\right)$, at most $-12.8 \mathrm{~cm}^{-1}$ deviation in wavenumber $\left(\mathrm{SiH}_{2} \mathrm{Se}\right)$, and at most $1.56 \mathrm{kcal} / \mathrm{mol}$ in integrated energy (GeHAs). The best performance is achieved in the case of changing $\mathrm{SiH}_{3} \mathrm{Cl} \rightarrow \mathrm{GeH}_{3} \mathrm{Br}$ with energy error $\Delta E=0.6 \mathrm{kcal} / \mathrm{mol}$ and integrated $\mathrm{MAE}=0.9 \mathrm{kcal} / \mathrm{mol}$. Corresponding predictions of equilibrium distance deviate $0.03 \AA$ with vibration frequency deviate $-1.1 \mathrm{~cm}^{-1}$. First order predictions do not 
yield quantitative predictive power for changes involving lighter elements (columns 2/3, 3/2, 2/4, 2/2, 4/2). The worst predictions are found for the simultaneous coupling to two lighter elements (column 2/2) with $76.29 \mathrm{kcal} / \mathrm{mol}, 0.35 \AA$, $-568.8 \mathrm{~cm}^{-1}$, and $41.45 \mathrm{kcal} / \mathrm{mol}$ deviation in minimum energy, bond length, harmonic frequency, and integrated energy ( $\mathrm{HCN})$.

While for all first order predictions all mutual deviations exhibit the same sign, second order corrections introduce the sign changes in minimum energy and bond length alluded to before, namely, both third row elements couple to lighter elements from the second row (column 2/2). Second order predictions for this column are even worse than the corresponding first order predictions. Second order predictions only improve first order predictions in the case of columns $2 / 3$ and $3 / 2$, alas, not to a degree considered satisfying.

In summary, for changes corresponding to columns $4 / 3$, $3 / 4,4 / 4$, first order based estimates yield chemical accuracy. For changes corresponding to columns $2 / 2$, first order based estimates are inaccurate but still better than second order estimates. For changes corresponding to columns $2 / 4$ and $4 / 2$, first order based estimates are similar to second order estimates, yet both are inaccurate. For changes corresponding to columns $2 / 3$ and $3 / 2$, second order based estimates are inaccurate but still better than first order estimates.

\section{Empirical $d_{\text {opt }}$}

The above observations have been made for optimized $d_{0}$. It should be noted that the choice of $d_{0}$ in Eq. (10) is crucial for linearizing the property of interest in alchemical coupling parameter $\lambda$ and hence essential for the performance of the perturbation based predictions. We have found that the error minimizing $d_{\text {opt }}$ has an approximately linear dependence on the target molecule's equilibrium bond length $d_{\text {eq }}$, no matter if the reference is the hydrogen containing single bond, or a single, double, or triple bond involving $p$-block elements from second, third, or fourth row. Furthermore, the linear relationship is preserved, independent of the fact if predictions are made with first or second order estimates. This relationship is shown in Fig. 3. The parameters of a linear regression are specified as well. The outlier in Fig. 3 at target $d_{\mathrm{eq}} \approx 1.35 \AA$ and $d_{\mathrm{opt}} \approx 1.0 \AA$ is due to the second order prediction of $\mathrm{SiH}_{3} \mathrm{Cl} \rightarrow \mathrm{CH}_{3} \mathrm{~F}$, i.e., for the above discussed worst case scenario (column 2/2) where a strong overcorrection has been found.

\section{Non-vertical iso-electronic changes}

We are not aware of any mathematical limitation on how to construct alchemical coupling paths under iso-electronic condition. In addition to the investigation of predicted PES of iso-electronic compounds with the same geometry, as discussed in Secs. III A and III B, we have also investigated if one can use only one reference calculation in order to estimate the entire PES through "non-vertical" interpolations. In other words, we have also assessed the applicability of the Taylor expansion of Eq. (9) to non-vertical changes for varying geometry and/or atom types and numbers between reference and target molecules.

\section{Alchemical stretching of $\mathrm{H}_{2}^{+}$}

We now turn to the case of alchemical stretching of $\mathrm{H}_{2}^{+}$in order to understand the effect of varying geometry on alchemical predictions. Since Hartree-Fock is formally exact for one-electron systems, we have employed an atomic basis set in an "all-electron" (no PPs) calculation within the following alignment scheme: One proton is centered at $\mathbf{R}_{1}=(0,0,0)$, and the other is aligned along the $+x$-axis. The reference system corresponds to $\mathrm{H}_{2}^{+}$at its equilibrium bond length. Stretching is accomplished not by pulling the atoms apart but rather by simultaneous annihilation and creation of nuclear charges at $\mathbf{R}_{2}^{\mathrm{R}}=\left(d_{\mathrm{eq}}, 0,0\right)$ and $\mathbf{R}_{2}^{\mathrm{T}}=(d, 0,0)$, respectively. Once the SCF is done for $d_{\text {eq }}$, the entire binding potential can be estimated up to $m=4$ order, using Eq. (11), by scanning through various $d$ 's, i.e., setting $d_{0}=d_{\text {eq }}$.

Results are shown in Fig. 5(a). Due to the variational principle for linearly coupled alchemical Hamiltonians, ${ }^{12}$ $\Delta E^{(1)}>\Delta E$ for all interatomic distances. Inclusion of second order term improves upon the first order prediction, yielding a reasonable binding potential. However, when including third and fourth order, the performance deteriorates again with oscillating behaviour for varying order (Fig. 5(a) and inset of Fig. 5(b)), as $\Delta E^{(3)}$ overshoot and $\Delta E^{(4)}$ over-corrects. Overall $\Delta E^{(2)}$ gives the best prediction.

To explain the oscillating behaviour in Taylor expansion order, we investigate in more detail how the system responds to alchemical perturbation. When $\lambda$ increases gradually from 0 to 1 , the nuclear charge decreases from 1 to 0 at $\mathbf{R}_{2}^{\mathrm{R}}$, while increasing from 0 to 1 at $\mathbf{R}_{2}^{\mathrm{T}}$. Using the alchemical derivatives at $\lambda=0$, truncated Taylor series based estimates are plotted along with the true energy in Fig. 5(b) as a function of $\lambda$ at $d=3 \AA$. $\Delta E^{(1)}, \Delta E^{(2)}, \Delta E^{(3)}$, and $\Delta E^{(4)}$ are linear, quadratic, third order, and fourth order polynomials, respectively. Clearly, the truncated Taylor series will fail to converge to $\Delta E$ at $\lambda=1$ due to a sharp change of $\Delta E$ at $\lambda \approx 0.9$. This implies a strong nonlinear electronic response occurring late in the alchemical coupling regime, resulting in the oscillating behaviour of the predicted PES in Figs. 5(a) and $5(b)$. Note that while the sign of error alternates, the magnitude of error also increases as one increases the order. Similar behaviour can be observed for other values of $d$.

The energy gain starting at $\lambda \approx 0.9$ is due to a rapid rearrangement of electron density for $\lambda>0.9$. This is illustrated in Fig. 5(c) where the integrated electron density $P_{\lambda}(x)$ is plotted as a function of both $\lambda$ and $x$ at $d=3 \AA$. Cohen and Mori-Sánchez already pointed out for $\mathrm{H}_{2}^{+}$the dramatic changes in electronic structure for infinitesimally small changes in nuclear charges at infinite distance. ${ }^{65}$ One would expect this effect to intensify as more basis functions are taken into account. This behaviour can be seen in Fig. 5(c). The locations of the proton at origin $\mathbf{R}_{1}$, the annihilated proton at $\mathbf{R}_{2}^{\mathrm{R}}$, and the created proton at $\mathbf{R}_{2}^{\mathrm{T}}$, are indicated by red lines.

Further analysis shows that for $\lambda>0.5$, both ground and first excited state orbitals are localized: The electronic ground 


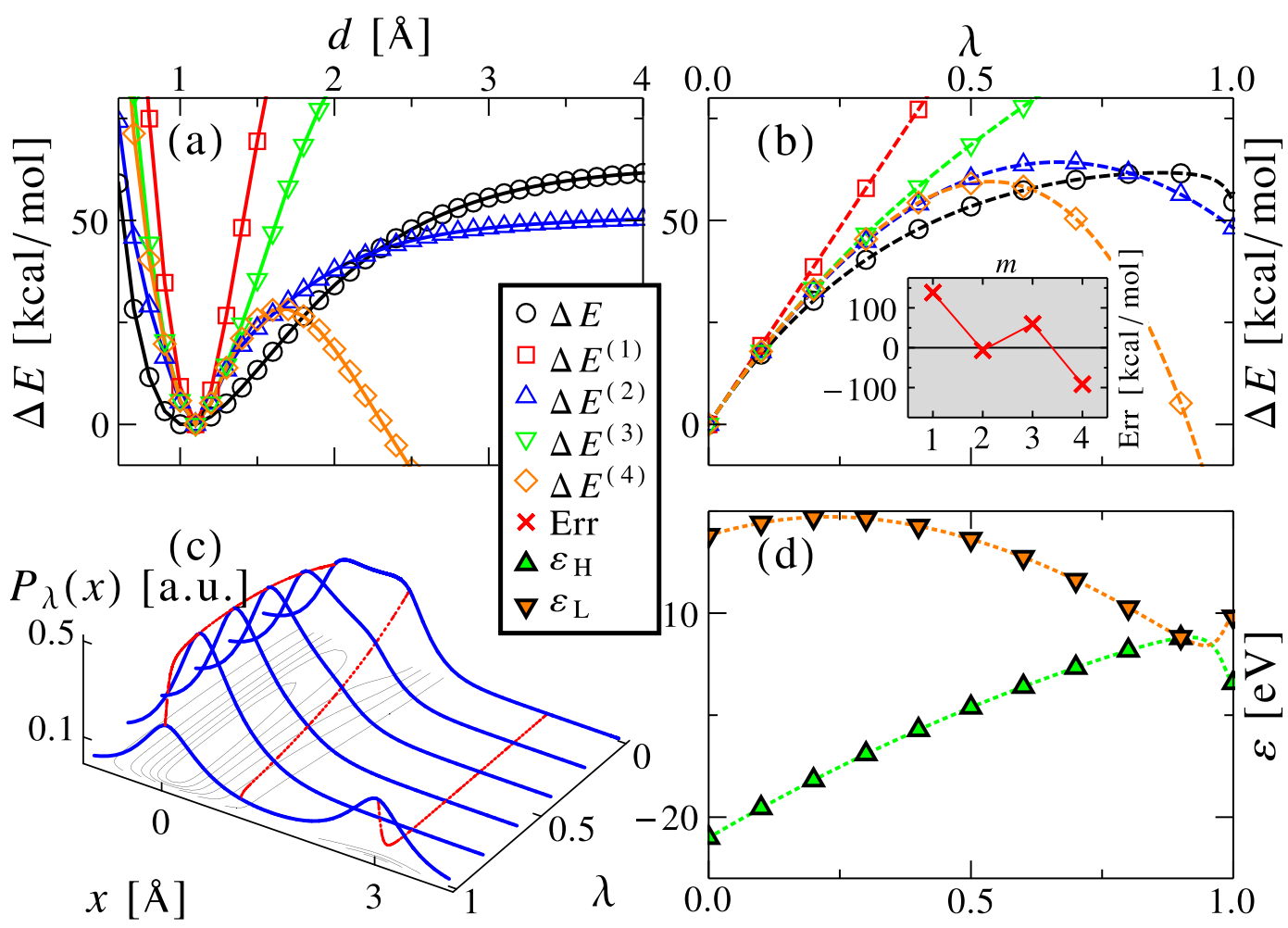

FIG. 5. $m$ th order truncated Taylor series of $\mathrm{H}_{2}^{+}$are denoted by $\Delta E^{(m)}$ in (a) as a function of $d$ at $\lambda=1$ and in (b) as a function of $\lambda$ at $d=3 \AA$. Inset shows the error of $\Delta E^{(m)}$ at $\lambda=1$. (c) Integrated density $P_{\lambda}(x)=\int d y d z \rho_{\lambda}(\mathbf{r})$, where $\mathbf{r}=(x, y, z)$, is presented as a function of both $\lambda$ and $x$ at $d=3 \AA$, where the integrated density values at nuclear locations are highlighted by red lines at $x=0 \AA$, $x=1.1 \AA$, and $x=3 \AA$, while contour lines are drawn at the bottom. (d) $\mathrm{HOMO} / \mathrm{LUMO}$ levels, denoted by $\varepsilon_{\mathrm{H}}$ and $\varepsilon_{\mathrm{L}}$, respectively, are plotted as a function of $\lambda$ at $d=3 \AA$.

state is localized at $\mathbf{R}_{1}$ while the first excited state is localized at $\mathbf{R}_{2}^{\mathrm{T}}$. At $\lambda \approx 0.9$, the two eigenvalues become degenerate, resulting in a rapid change of the ground state density in order to meet the non-polar symmetry requirement of $\mathrm{H}_{2}^{+}$, by fluctuating between both ground and first excited states. Note that there is no orbital node at midpoint, indicating a true ground state for a dissociated $\mathrm{H}_{2}^{+}$molecule. The degeneracy occurs for the system with fractional nuclear charges at $\lambda \approx 0.9$. The dramatic change in density stabilizes the system in $\lambda$, giving rise to the sharp decrease in energy in Fig. 5(b), as $\lambda$ increases from 0.8 to 1 . Perturbation theory for degenerate cases might be necessary to properly account for this case. The degeneracy of the ground state and first excited state is shown for the eigenvalue crossing in Fig. 5(d): The eigenvalues of the (highest) occupied molecular orbital (HOMO) and lowest unoccupied molecular orbital (LUMO) are plotted as a function of $\lambda$. The degeneracy breaks when ground state and first excited state switch order, which results in a delocalized ground state. By contrast, note that the eigenvalues will not cross each other if the stretching is carried out by moving $\mathbf{R}_{2}^{\mathrm{R}}$ in real space.

Crossing of eigenvalue surfaces limits the radius of convergence of alchemical Taylor expansion series within electronic ground-state theories. As a result, the Taylor expansion for this system is not convergent at $\lambda=1$, similar to well-known cases in Møller-Plesset theory. ${ }^{66-68}$ For asymmetric alchemical interpolations, as exemplified for the following examples in this study, as well as in previous studies, ${ }^{11,17,20,32}$ the energy is typically smooth in all $\lambda$ values, and derivative based expansions are expected to converge.

\section{Non-vertical iso-electronic changes in ten electron systems}

Now we consider alchemical non-vertical changes of molecules with ten electrons. More specifically, we present numerical results of non-vertical iso-electronic changes involving bond stretching in second row systems $\left\{\mathrm{CH}_{4}, \mathrm{NH}_{3}\right.$, $\left.\mathrm{H}_{2} \mathrm{O}, \mathrm{HF}\right\}$, using all electron DFT. First order estimates have been obtained according to Eq. (15). The $\mathrm{H}_{2}^{+}$example has indicated that non-vertical changes can profit from second order estimates. Since exact analytical expressions are not available for systems with so many electrons, and since these changes do not require changing pseudopotentials, we have relied for this section on the IPA and CP second order expressions, rather than on finite difference expressions (see Sec. II).

\section{Predicted potentials}

Fig. 6 illustrates the prediction of R-H covalent bond potentials for $\mathrm{CH}_{4}$ and $\mathrm{NH}_{3}$, predicted from alchemical derivatives using the electronic structure obtained by a single SCF. As a reference system we used once the relaxed $\mathrm{CH}_{4}$ system (panels (a) and (c)), and once the relaxed $\mathrm{NH}_{3}$ (panels (b) and (d)) geometry. For the chemical composition of $H_{\mathrm{R}}$ being the same as $H_{\mathrm{T}}$ and only the bond being stretched (Figs. 6(a) and 6(d)), the first order estimate constitutes an upper bound, i.e., it always overshoots due to the concave behaviour of $\Delta E$ as a function of $\lambda$, also on display in Fig. 5(b). When also changing the chemical compositions 


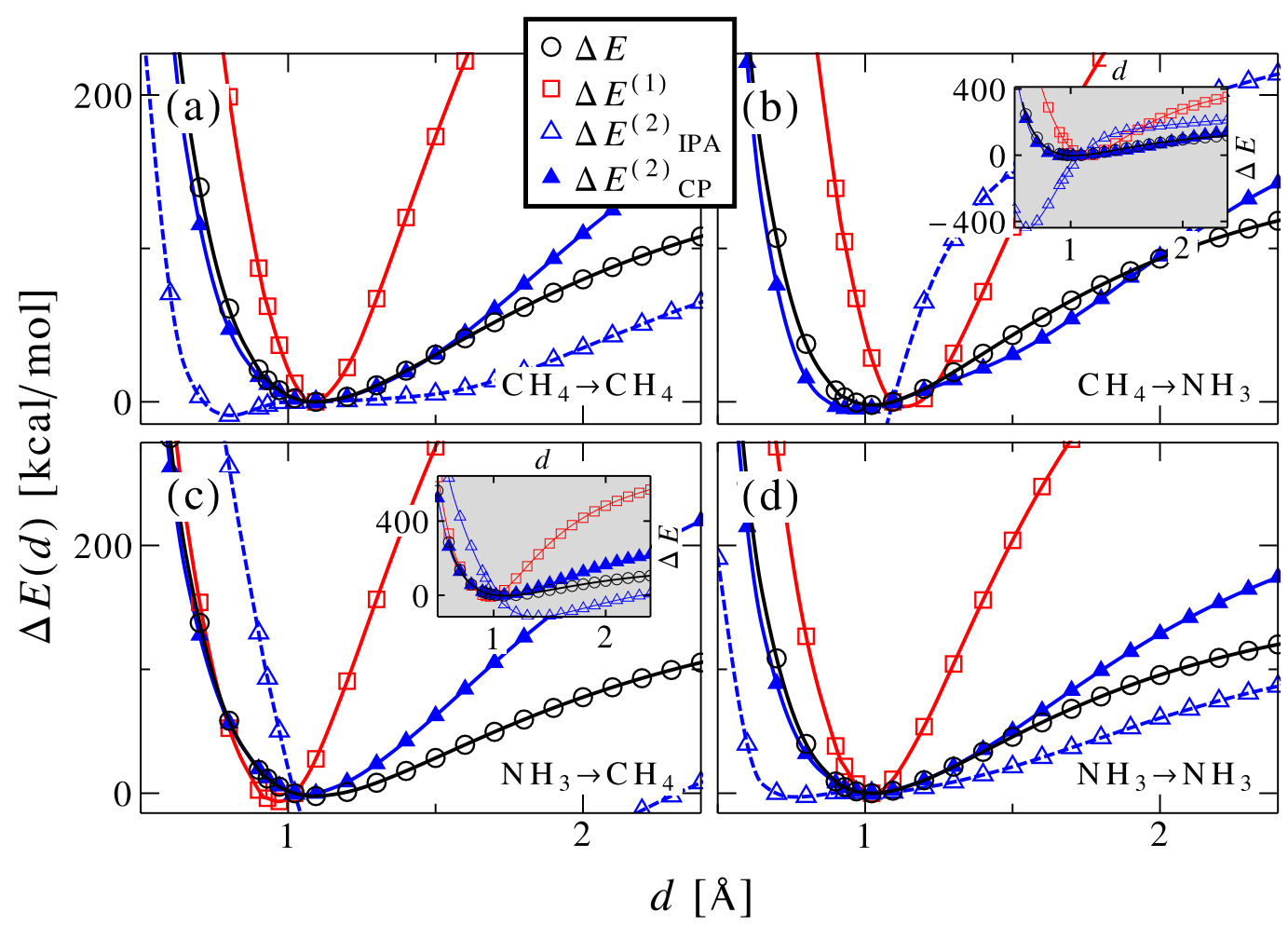

FIG. 6. N-H and C-H covalent bond potentials in methane and ammonia. Energy difference $\Delta E$, first order truncated Taylor series $\Delta E^{(1)}$, second order truncated Taylor series calculated by coupled perturbed $\Delta E_{\mathrm{CP}}^{(2)}$, and second order truncated Taylor series calculated by independent particle approximation $\Delta E_{\mathrm{IPA}}^{(2)}$ are plotted as black circles, red squares, blue open triangles, and blue filled triangles, respectively. Coupling Hamiltonians are arranged as follows: (a) $\mathrm{CH}_{4} \rightarrow \mathrm{CH}_{4}$, (b) $\mathrm{NH}_{3} \rightarrow \mathrm{CH}_{4}$, (c) $\mathrm{CH}_{4} \rightarrow \mathrm{NH}_{3}$, and (d) $\mathrm{NH}_{3} \rightarrow \mathrm{NH}_{3}$. Insets of (b) and (c) show the zoom-out energy scale for overall landscape.

from $\mathrm{CH}_{4}$ to $\mathrm{NH}_{3}$ or vice versa, the first order estimate does not even capture the changes in equilibrium bond length (Figs. 6(b) and 6(c)).

$\Delta E_{\mathrm{IPA}}^{(2)}$ yields a saddle point in Figs. 6(a) and 6(d), instead of a minimum at optimized geometry. When the chemical compositions of $H_{\mathrm{R}}$ and $H_{\mathrm{T}}$ are different, $\Delta E_{\mathrm{IPA}}^{(2)}$ results in dramatic errors (worse than first order estimates), as shown in the energy zoom out in the insets of Figs. 6(b) and 6(c). The poor predictively power of IPA has also recently been pointed out by Pulay and co-workers. ${ }^{69}$ By contrast, $\Delta E_{\mathrm{CP}}^{(2)}$ yields a very reasonable binding potential, albeit still far from being chemically accurate. The superior performance of $\Delta E_{\mathrm{CP}}^{(2)}$, with respect to $\Delta E_{\mathrm{IPA}}^{(2)}$, indicates that the contributions of Coulomb and $\mathrm{xc}$ energy due to density response are crucial. In other words, matrix elements $\mathbf{J}_{i a, j b}$ and $\mathbf{X}_{i a, j b}$ in Eq. (8) should not be neglected for non-vertical alchemical perturbations.

Different predictive accuracy is found for compressing bonds $d<d_{\text {eq }}$ versus stretching bonds $d>d_{\text {eq }} . \Delta E_{\mathrm{CP}}^{(2)}$ performs better in the region $0.5 \AA \leq d \leq 1.5 \AA$. Similar behaviour is also observed for other alchemical paths of compressing vs stretching bond. Also in this case, the aforementioned non-commutative asymmetric behavior of the predictions is observed. Namely, the $\Delta E_{\mathrm{CP}}^{(2)}$ based prediction for $\mathrm{CH}_{4} \rightarrow \mathrm{NH}_{3}$ in Fig. 6(c) is more accurate than for $\mathrm{NH}_{3} \rightarrow \mathrm{CH}_{4}$ in Fig. 6(b). Note that abrupt changes in electronic structure, as observed for $\mathrm{H}_{2}^{+}$in Sec. III D 1, are not present when coupling these systems. ${ }^{70,71}$ Since the accuracy of the second order estimate is determined by how linearly the electron density rearranges as a function of $\lambda$, one expects a near-constant $\partial_{\lambda} \rho$ for negligible higher order contributions. This is confirmed through inspection of the integrated density response of the alchemical path $\mathrm{HF} \rightarrow \mathrm{H}_{2} \mathrm{O}$ in Fig. 7. $\partial_{\lambda} P_{\lambda}(x)$ varies less when $\lambda$ changes from zero to one for $d=0.5 \AA$ in Fig. 7(a), when compared with $d=1.5 \AA$ in Fig. 7(b). A near constant $\partial_{\lambda} P_{\lambda}(x)$ at $d=0.5 \AA$ results in improved predictive accuracy.

\section{Integrated errors}

Table SIII $^{72}$ summarizes the results for all $4 \times 4$ combinations of $H_{\mathrm{R}} \rightarrow H_{\mathrm{T}}$, where mutual predictions of covalent bond potentials in $H_{\mathrm{T}}:\left\{\mathrm{CH}_{4}, \mathrm{NH}_{3}, \mathrm{H}_{2} \mathrm{O}, \mathrm{HF}\right\}$ are obtained based on only the single point wavefunctions obtained for the relaxed geometry of $H_{\mathrm{R}}:\left\{\mathrm{CH}_{4}, \mathrm{NH}_{3}\right.$, $\left.\mathrm{H}_{2} \mathrm{O}, \mathrm{HF}\right\}$, respectively. This coupling matrix in chemical space is not symmetric due to the non-commutative properties discussed above. Off-diagonal elements correspond to coupling paths involving changes in chemical composition and geometry. Diagonal elements correspond to coupling paths that involve only changes in geometry, i.e., for the same stoichiometry. Note that all error measures have been obtained via cubic spline fits. Therefore, also the predicted $\Delta E$ and the location of the energy minimum can be slightly non-zero even for the diagonal elements. These values should be considered as noise: for the diagonal elements only the harmonic frequencies are meaningful.

Results in Table SIII ${ }^{72}$ confirm the trends observed above for first and second order. In general, best predictive power is found when the chemical composition of $H_{\mathrm{R}}$ is the same as $H_{\mathrm{T}}$ (diagonal elements). When the chemical composition of $H_{\mathrm{T}}$ differs from $H_{\mathrm{R}}$, the predictive accuracy deteriorates. This 


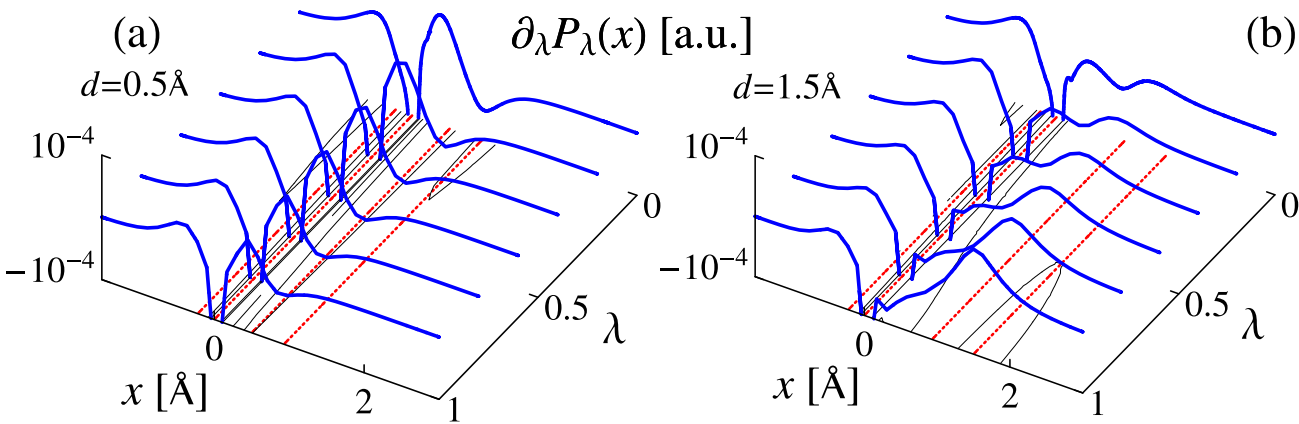

FIG. 7. Response of integrated slices of electron density (see Fig. 5). $\partial_{\lambda} P_{\lambda}(x)$ is calculated by finite difference $\partial_{\lambda} P_{\lambda}(x) \approx \frac{P_{\Delta \lambda}(x)-P_{\lambda=0}(x)}{\Delta \lambda}$. $\partial_{\lambda} P_{\lambda}(x)$ of $\mathrm{HF} \rightarrow \mathrm{H}_{2} \mathrm{O}$ at (a) $d=0.5 \AA$ for compression and at (b) $d=1.5 \AA$ for extension are plotted as a function of $x$ and $\lambda$. Nuclear positions are highlighted by red dashed lines, with $\mathrm{F} \rightarrow \mathrm{O}$ at $x=0 \AA, \mathrm{H} \rightarrow$ void at $x=0.93 \AA$, two void $\rightarrow \mathrm{H}$ at $x=-0.22 \AA$ and $x=d$, where void denotes the nuclei with zero charge.

is not surprising and due to the perturbing Coulomb potential being placed on the heavy atom in order to mutate it, e.g., from carbon to fluorine. Because of the strong accumulation of electron density (cusps) at the heavy atom's site (6 to 9 electrons for carbon to fluorine, respectively), this perturbation is quite severe. In the case of the diagonal element, by contrast, only the hydrogen atom is being annihilated and created, implying that the perturbing potential acts on the hydrogen atom's electronic density which is built up by only 1 electron. This implies a less severe perturbation, and therefore, worse predictive power can be expected for off-diagonal elements.

The crucial importance of Coulomb and $\mathrm{xc}$ energy contribution to density response for second order alchemical perturbation is also confirmed for the other cases in Table SIII. ${ }^{72}$ These results clearly underscore the observation that IPA is a (very) poor approximation when it comes to estimate alchemical changes, yielding even worse predictions than the first order estimates. Interestingly enough, the first order estimate is even competitive in comparison to the second order $\mathrm{CP}$ predictions. For example, using $\mathrm{CH}_{4}$ as a reference compound, the first order prediction deviates on average by $-8.54 \mathrm{kcal} / \mathrm{mol}$ in the energy, while $\Delta E_{\mathrm{CP}}^{(2)}$ deviates by $-15.99 \mathrm{kcal} / \mathrm{mol}$. However, as the reference compound moves to the right hand side of the periodic table, the second order $\mathrm{CP}$ based estimate becomes more accurate than the first order based estimate.

An additional aspect can be confirmed from inspection of Table SIII: ${ }^{72}$ the larger the perturbing potential, the worse the predictive accuracy of derivative based estimates. More specifically, the larger the integrated norm of the difference between reference and target potentials in the electronic Hamiltonian, the worse the predictive power. For example, using $\mathrm{CH}_{4}$ as a reference compound, the prediction will be increasingly worse in the order of the respective predictions for $\mathrm{NH}_{3}, \mathrm{H}_{2} \mathrm{O}$, and $\mathrm{HF}$. Conversely, using $\mathrm{HF}$ as a reference compound, the prediction will be increasingly worse in the order of the respective predictions for $\mathrm{H}_{2} \mathrm{O}, \mathrm{NH}_{3}$, and $\mathrm{CH}_{4}$. Note that this is true for all first as well as second order estimates.

\section{CONCLUSIONS}

The performance of truncated Taylor series for predicting alchemical vertical changes in covalent bonding has been investigated in iso-electronic chemical spaces spanned by the external potentials of small molecules. For vertical linear transmutations (same geometry, same number of atoms, differing nuclear charges), our results suggest that chemical accuracy is possible when interpolating molecules containing $p$-block atoms from the third and fourth row using first order (Hellmann-Feynman theory) based predictions. Since first order estimates are analytical, this finding implies that one can scan potential energy surfaces of very many molecules with unprecedented accuracy and speed as long as their stoichiometries are restricted to third and fourth row main group chemistries. First order based predictions of chemistries involving second row elements are only correct to a degree considered qualitative.

Overall, we have found second order estimates to provide insufficient improvement with respect to first order predictions (often even worse) to warrant the investment in the additional overhead incurred. First order estimates are more accurate not because higher order terms are negligible, but rather due to the fact that (a) changes in relative energies (bonding) are already near-linear (by optimizing the reference geometry) with respect to alchemical coupling (effectively canceling higher order terms), and (b) inflection points can occur which lead to worse predictions for second order estimates. For the interpolation of the pseudopotentials used in this study, inflection points near $\lambda=0$ are always observed when a lighter main group element is coupled to a heavier one. The absence of inflection points near $\lambda=1$ improves the predictive power of the second order correction when coupling a heavier element to a lighter one. As such, the asymmetry of $\Delta E\left(d, d_{0}\right)$ with respect to $\lambda=0.5$ results in asymmetric predictive performance.

The choice of reference geometry has a dramatic impact on the predictive power of the alchemical estimates. For covalent bond potentials, a linear relationship has been identified $\left(d_{\text {opt }} \approx 0.76 d_{\mathrm{eq}}^{\mathrm{T}}+0.97 \AA\right)$ that can be used to predict optimal $d_{0}$ requiring only rough estimates of the equilibrium bond-length in the target molecule (which can be obtained with small computational overhead, using universal force-fields or semi-empirical quantum chemistry methods).

We have found oscillating behaviour in the predictions of truncated Taylor series with increasing order when considering non-vertical alchemical stretching of $\mathrm{H}_{2}^{+}$. The crossing of eigenvalue surfaces is due to the electron density's necessity 
to be symmetric at $\lambda=0$ and $\lambda=1$. This leads to a diverging Taylor series, but the second order correction can still provide fair predictions. The behavior of first and second order truncated alchemical Taylor series expansions in non-vertical transmutations in chemical space has also been analyzed for molecules with ten electrons. Numerical evidence of the superior performance of $\Delta E_{\mathrm{CP}}^{(2)}$ over $\Delta E_{\mathrm{IPA}}^{(2)}$ suggests that the response of Coulomb and xc energy is crucial to alchemical perturbation.

In summary, our findings indicate that a careful choice of alchemical interpolation paths enables alchemical derivatives to achieve predictive power with chemical accuracy for covalent bond potentials. Future work will deal with angles and torsions in larger molecules, as well as with solid metals and ionic crystals.

\section{ACKNOWLEDGMENTS}

We would like to thank K. Morokuma, E. Tapavicza, Q. Cui, A. Alavi, P. Ayers, and P. Geerlings for discussions. O.A.v.L. acknowledges funding from the Swiss National Science Foundation (No. PP00P2_138932). S.F. acknowledges the Research Foundation Flanders (FWO) for financial support. Some calculations were performed at sciCORE (http://scicore.unibas.ch/) scientific computing core facility at University of Basel.

${ }^{1}$ E. Schrödinger, "An undulatory theory of the mechanics of atoms and molecules," Phys. Rev. 28, 1049 (1926).

${ }^{2}$ P. A. M. Dirac, "Quantum mechanics of many-electron systems," Proc. Math. Phys. Eng. Sci. 123, 714 (1929).

${ }^{3}$ J. Harris, "Simplified method for calculating the energy of weakly interacting fragments," Phys. Rev. B 31, 1770 (1985).

${ }^{4}$ J. Harris, "Tight-binding models and density-functional theory," Phys. Rev. B 39, 12520 (1989).

${ }^{5}$ D. Benoit, D. Sebastiani, and M. Parrinello, "Accurate total energies without self-consistency," Phys. Rev. Lett. 87, 226401 (2001).

${ }^{6}$ S. R. Marder, D. N. Beratan, and L. T. Ceng, "Approaches for optimizing the first electronic hyperpolarizability of conjugated organic molecules," Science 252, 103 (1991).

${ }^{7}$ A. Franceschetti and A. Zunger, "The inverse band-structure problem of finding an atomic configuration with given electronic properties," Nature 402, 60 (1999).

${ }^{8}$ G. H. Jóhannesson, T. Bligaard, A. V. Ruban, H. L. Skriver, K. W. Jacobsen, and J. K. Nørskov, "Combined electronic structure and evolutionary search approach to materials design," Phys. Rev. Lett. 88, 255506 (2002).

${ }^{9} \mathrm{O}$. A. von Lilienfeld, in Towards the Computational Design of Compounds from First Principles, Mathematical Physics Studies Vol. IX, edited by V. Bach and L. Delle Site (Springer, 2014).

${ }^{10}$ T. F. T. Cerqueira, R. Sarmiento-Prez, M. Amsler, F. Nogueira, S. Botti, and M. A. L. Marques, "Materials design on-the-fly," J. Chem. Theory Comput. 11(8), 3955-3960 (2015)

${ }^{11}$ O. A. von Lilienfeld and M. E. Tuckerman, "Molecular grand-canonical ensemble density functional theory and exploration of chemical space," J. Chem. Phys. 125, 154104 (2006).

${ }^{12}$ O. A. von Lilienfeld, "First principles view on chemical compound space: Gaining rigorous atomistic control of molecular properties," Int. J. Quantum Chem. 113, 1676 (2013).

${ }^{13}$ C. Kuhn and D. N. Beratan, "Inverse strategies for molecular design," J. Phys. Chem. 100, 10595 (1996).

${ }^{14}$ M. E. M. Noble, J. A. Endicott, and L. N. Johnson, "Protein kinese inhibitors: Insights into drug design from structure," Science 303, 1800 (2004).

${ }^{15}$ S. Keinan, X. Hu, D. Beratan, and W. Yang, "Designing molecules with optimal properties using the linear combination of atomic potentials approach in an AM1 semiempirical framework," J. Phys. Chem. 111, 176 (2007).

${ }^{16}$ R. Car and M. Parrinello, "Unified approach for molecular dynamics and density functional theory," Phys. Rev. Lett. 55, 2471 (1985).
${ }^{17}$ O. A. von Lilienfeld, R. D. Lins, and U. Rothlisberger, "Variational particle number approach for rational compound design," Phys. Rev. Lett. 95, 153002 (2005).

${ }^{18}$ M. Wang, X. Hu, D. N. Beratan, and W. Yang, "Designing molecules by optimizing potentials," J. Am. Chem. Soc. 128, 3228 (2006).

${ }^{19}$ V. Marcon, O. A. von Lilienfeld, and D. Andrienko, "Tuning electronic eigenvalues of benzene via doping," J. Chem. Phys. 127, 064305 (2007).

${ }^{20} \mathrm{O}$. A. von Lilienfeld, "Accurate $a b$ initio energy gradients in chemical compund space," J. Chem. Phys. 131, 164102 (2009).

${ }^{21}$ D. Sheppard, G. Henkelman, and O. A. von Lilienfeld, "Alchemical derivatives of reaction energetics," J. Chem. Phys. 133, 084104 (2010).

${ }^{22} \mathrm{~T}$. Weymuth and M. Reiher, "Inverse quantum chemistry: Concepts and strategies for rational compound design," Int. J. Quantum Chem. 114, 823 (2014).

${ }^{23}$ B. Widom, "Some topics in the theory of fluids," J. Chem. Phys. 39(11), 2808-2812 (1963)

${ }^{24}$ M. D. Macedonia and E. J. Maginn, "A biased grand canonical Monte Carlo method for simulating adsorption using all-atom and branched united atom models," Mol. Phys. 96, 1375 (1999).

${ }^{25} \mathrm{M}$. D. Macedonia and E. J. Maginn, "Pure and binary component sorption equilibria of light hydrocarbons in the zeolite silicalite from grand canonical Monte Carlo simulations," Fluid Phase Equilib. 158-160, 19 (1999).

${ }^{26} \mathrm{~W}$. L. Jorgensen and C. Ravimohan, "Monte Carlo simulation of differences in free energies of hydration," J. Chem. Phys. 83, 3050 (1985).

${ }^{27}$ W. G. van Gunsteren and H. J. C. Berendsen, "Thermodynamic cycle integration by computer simulation as a tool for obtaining free energy differences in molecular chemistry," J. Comput.-Aided Mol. Des. 1, 171 (1987).

${ }^{28}$ T. Straatsma and J. A. McCammon, "Computational alchemy," Annu. Rev. Phys. Chem. 43, 407 (1992).

${ }^{29}$ C. Oostenbrink and W. F. van Gunsteren, "Free energies of ligand binding for structurally diverse compounds," Proc. Natl. Acad. Sci. U. S. A. 102, 6750 (2005).

${ }^{30}$ S. Riniker, C. D. Christ, H. S. Hansen, P. H. Hünenberger, C. Oostenbrink, D. Steiner, and W. F. van Gunsteren, "Calculation of relative free energies for ligand-protein binding, solvation, and conformational transitions using the GROMOS software," J. Phys. Chem. B 115, 13570 (2011).

${ }^{31}$ J. E. Bright Wilson, "Four-dimensional electron density function," J. Phys. Chem. 36, 2232 (1962).

${ }^{32}$ K. Y. S. Chang and O. A. von Lilienfeld, "Quantum mechanical treatment of variable molecular composition: From 'alchemical' changes of state function to rational compound design," CHIMIA 68, 602 (2014).

${ }^{33}$ R. P. Feynman, "Forces in molecules," Phys. Rev. 56, 340 (1939).

${ }^{34} \mathrm{O}$. A. von Lilienfeld and M. E. Tuckerman, "Alchemical variations of intermolecular energies according to molecular grand-canonical ensemble density functional theory," J. Chem. Theory Comput. 3, 1083 (2007).

${ }^{35}$ R. Balawender, M. A. Welearegay, M. Lesiuk, F. De Proft, and P. Geerlings, "Exploring chemical space with the alchemical derivatives," J. Chem. Theory Comput. 9, 5327 (2013)

${ }^{36} \mathrm{E}$. K. U. Gross and W. Kohn, "Local density-functional theory of frequency dependent linear response," Phys. Rev. Lett. 55, 2850 (1986).

${ }^{37}$ W. Yang, A. J. Cohen, F. De Proft, and P. Geerlings, "Analytical evaluation of Fukui function and real space linear response function," J. Chem. Phys. 136, 144110 (2012).

${ }^{38}$ N. Sablon, F. De Proft, P. W. Ayers, and P. Geerlings, "Computing secondorder functional derivatives with respect to the external potential," J. Chem. Theory Comput. 6, 3671 (2010).

${ }^{39}$ R. G. Parr and W. Yang, Density-Functional Theory of Atoms and Molecules (Oxford University Press, USA, 1994).

${ }^{40}$ A. Putrino, D. Sebastiani, and M. Parrinello, "Generalized variational density functional perturbation threory," J. Chem. Phys. 113, 7102 (2000).

${ }^{41}$ H. F. Wilson, F. Gygi, and G. Galli, "Efficient iterative method for calculations of dielectric matrices," Phys. Rev. B 78, 113303 (2008).

${ }^{42}$ N. Sablon, F. De Proft, and P. Geerlings, "The linear response kernel: Inductive and resonance effects quantified," J. Phys. Chem. Lett. 1, 1227 (2010).

${ }^{43}$ S. Fias, Z. Boisenghien, T. Stuyver, M. Audiffred, G. Merino, P. Geerlings, and F. De Proft, "Analysis of aromaticity in planar metal systems using the linear response kernel," J. Phys. Chem. A 117, 3556 (2013).

${ }^{44}$ P. Geerlings, S. Fias, Z. Boisdenghen, and F. De Proft, "Conceptual DFT: Chemistry from the linear response function," Chem. Soc. Rev. 43, 4989 (2014).

${ }^{45}$ S. Baroni, S. de Gironcoli, A. Dal Corso, and P. Giannozzi, "Phonons and related crystal properties from density-functional perturbation theory," Rev. Mod. Phys. 73, 515-562 (2001). 
${ }^{46}$ S. L. Adler, "Quantum theory of the dielectric constant in real solids," Phys. Rev. 126(2), 413 (1962).

${ }^{47} \mathrm{~N}$. Wiser, "Dielectric constant with local field effects included," Phys. Rev. 129(1), 62 (1963).

${ }^{48} \mathrm{C}$. Møller and M. S. Plesset, "Note on an approximation treatment for manyelectron systems," Phys. Rev. 46, 618 (1934).

${ }^{49}$ D. Cremer, "Møller-Plesset perturbation theory: From small molecule methods to methods for thousands of atoms," Wiley Interdisc. Rev.: Comput. Mol. Sci. 1, 509 (2011).

${ }^{50}$ L. Kleinman and D. M. Bylander, "Efficient form for model pseudopotentails," Phys. Rev. Lett. 48, 1425 (1982).

${ }^{51}$ O. A. von Lilienfeld, I. Tavernelli, U. Rothlisberger, and D. Sebastiani, "Optimization of effective atom centered potentials for London dispersion forces in density functional theory," Phys. Rev. Lett. 93, 153004 (2004).

${ }^{52} \mathrm{O}$. A. von Lilienfeld and P. A. Schultz, "Structure and band gaps of Ga-(V) semiconductors: The challenge of Ga pseudopotentials," Phys. Rev. B 77, 115202 (2008).

${ }^{53}$ O. A. von Lilienfeld, "Force correcting atom centred potentials for generalised gradient approximated density functional theory: Approaching hybrid functional accuracy for geometries and harmonic frequencies in small chlorofluorocarbons," Mol. Phys. 111(14-15), 2147-2153 (2013).

${ }^{54}$ J. P. Perdew, K. Burke, and M. Ernzerhof, "Generalized gradient approximation made simple," Phys. Rev. Lett. 77, 3865 (1996).

${ }^{55} \mathrm{~W}$. Andreoni and A. Curioni, "New advances in chemistry and materials science with CPMD and parallel computing," Parallel Comput. 26, 819-842 (2000).

${ }^{56}$ S. Goedecker, M. Teter, and J. Hutter, "Separable dual-space Gaussian pseudopotentials," Phys. Rev. B 54, 1703 (1996).

${ }^{57} \mathrm{C}$. Hartwigsen, S. Goedecker, and J. Hutter, "Relativistic separable dualspace Gaussian pseudopotentials from H to Rn," Phys. Rev. B 58, 3641 (1998).

${ }^{58} \mathrm{M}$. Krack, "Pseudopotentials for $\mathrm{H}$ to $\mathrm{Kr}$ optimized for gradient-corrected exchange-correlation functionals," Theor. Chim. Acta 114, 145 (2005).

${ }^{59}$ M. J. Frisch, G. W. Trucks, H. B. Schlegel, G. E. Scuseria, M. A. Robb, J. R. Cheeseman, G. Scalmani, V. Barone, B. Mennucci, G. A. Petersson, H. Nakatsuji, M. Caricato, X. Li, H. P. Hratchian, A. F. Izmaylov, J. Bloino, G. Zheng, J. L. Sonnenberg, M. Hada, M. Ehara, K. Toyota, R. Fukuda, J. Hasegawa, M. Ishida, T. Nakajima, Y. Honda, O. Kitao, H. Nakai, T. Vreven, J. A. Montgomery, Jr., J. E. Peralta, F. Ogliaro, M. Bearpark, J. J. Heyd, E. Brothers, K. N. Kudin, V. N. Staroverov, R. Kobayashi, J. Normand, K. Raghavachari, A. Rendell, J. C. Burant, S. S. Iyengar, J. Tomasi, M. Cossi,
N. Rega, J. M. Millam, M. Klene, J. E. Knox, J. B. Cross, V. Bakken, C. Adamo, J. Jaramillo, R. Gomperts, R. E. Stratmann, O. Yazyev, A. J. Austin, R. Cammi, C. Pomelli, J. W. Ochterski, R. L. Martin, K. Morokuma, V. G. Zakrzewski, G. A. Voth, P. Salvador, J. J. Dannenberg, S. Dapprich, A. D. Daniels, Ö. Farkas, J. B. Foresman, J. V. Ortiz, J. Cioslowski, and D. J. Fox, GaUSSIAN 09, Revision D.01, Gaussian, Inc., Wallingford, CT, 2009.

${ }^{60} \mathrm{~T}$. H. Dunning, Jr., "Gaussian basis sets for use in correlated molecular calculations. I. The atoms boron through neon and hydrogen," J. Chem. Phys. 90, 1007 (1989).

${ }^{61}$ M. Valiev, E. Bylaska, N. Govind, K. Kowalski, T. Straatsma, H. van Dam, D. Wang, J. Nieplocha, T. W. E. Apra, and W. de Jong, "NWChem: A comprehensive and scalable open-source solution for large scale molecular simulations," Comput. Phys. Commun. 181, 1477 (2010).

${ }^{62} \mathrm{~F}$. Weigend and R. Ahlrichs, "Balanced basis sets of split valence, triple zeta valence and quadruple zeta valence quality for $\mathrm{H}$ to $\mathrm{Rn}$ : Design and assessment of accuracy," Phys. Chem. Chem. Phys. 7, 3297 (2005).

${ }^{63}$ C. Adamo, M. Cossi, G. Scalmani, and V. Barone, "Accurate static polarizabilities by density functional theory: Assessment of the PBE0 model," Chem. Phys. Lett. 307, 265 (1999).

${ }^{64} \mathrm{~K}$. Burke, "Perspective on density functional theory," J. Chem. Phys. 136, 150901 (2012).

${ }^{65}$ A. J. Cohen and P. Mori-Sánchez, "Dramatic changes in electronic structure revealed by fractional charged nulei," J. Chem. Phys. 140, 044110 (2014).

${ }^{66}$ A. V. Sergeev, D. Z. Goodson, S. E. Wheele, and W. D. Allen, "On the nature of the Møller-Plesset critical point," J. Chem. Phys. 123, 064105 (2005).

${ }^{67}$ M. L. Leininger, W. D. Allen, H. F. Schaefer III, and C. D. Sherrill, "Is Møller-Plesset perturbation theory a convergent $a b$ initio method?," J. Chem. Phys. 112, 9213 (2000).

${ }^{68} \mathrm{~F}$. H. Stillinger, "Møller-Plesset convergence issues in computational quantum chemistry," J. Chem. Phys. 112, 9711 (2000).

${ }^{69}$ T. Janowski, K. Wolinski, and P. Pulay, "Efficient calculation of the density response function from generalized polarizabilities," Theor. Chim. Acta 135, 6 (2016).

${ }^{70}$ P. Mori-Sánchez, A. J. Cohen, and W. Yang, "Localization and delocalization errors in density functional theory and implication for band-gap prediction," Phys. Rev. Lett. 100, 146401 (2008).

${ }^{71}$ A. J. Cohen, P. Mori-Sánchez, and W. Yang, "Challenges for density functional theory," Chem. Rev. 112, 289 (2012).

${ }^{72}$ See supplementary material at http://dx.doi.org/10.1063/1.4947217 for tables with numerical results, as well as additional illustrative figures. 